\title{
Near-ultraviolet to near-infrared spectral properties of hollows on Mercury: Implications for origin and formation process
}

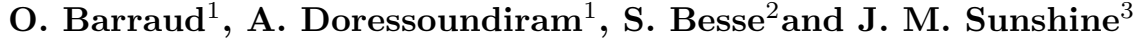 \\ ${ }^{1}$ LESIA, Observatoire de Paris, Université PSL, CNRS, Sorbonne Université, Université de Paris, 92195 \\ Meudon, France \\ ${ }^{2}$ European Space Astronomy Centre, Camino Bajo del Castillo s/n, 28692 Villanueva de la Canada, \\ Madrid, Spain \\ ${ }^{3}$ Department of Astronomy, University of Maryland, College Park, MD 20742-2421, USA \\ Key Points: \\ - Hollows have unique spectral properties in the near-ultraviolet. \\ - The reflectance spectra of hollows have a pronounced curvature between 300 and \\ $600 \mathrm{~nm}$. \\ - Eminescu impact crater hollows are seen to grow via scarp retreat.
}

Corresponding author: Océane Barraud, oceane.barraud@obspm.fr 


\begin{abstract}
[Among the geological features revealed by the MESSENGER (MErcury Surface, Space ENvironment, GEochemistry and Ranging) mission on the surface of Mercury, hollows are the most surprising and least understood. Possibly related to volatile components, hollows are small depressions, surrounded by bright halos and are not observed on any other surfaces in our Solar System. Previous analysis of multi-spectral data obtained by Mercury Dual Imaging System (MDIS) has shown that some hollows have weak spectral absorption features centered at around $600 \mathrm{~nm}$. In this work, we analyzed four hollows with observations acquired by the Mercury Atmospheric and Surface Composition Spectrometer (MASCS) on board MESSENGER with more than 230 spectral channels from the near-ultraviolet to the near-infrared. Unlike previous MDIS multispectral data, the MASCS reflectance spectra exhibit no absorption features in the MDIS wavelength range. However, we found that hollows have unique spectral properties in the near-ultraviolet, with a spectral curvature between 300 and $600 \mathrm{~nm}$ that is distinctly different from other geological units. Moreover, we used MASCS observations with the best available spatial resolution (less than $0.5 \mathrm{~km} / \mathrm{pixel}$ ) to analyze both parts of a hollow: the flat floor and the surrounding halo. Our results support the hypothesis that hollows form by a sublimation process and scarp retreat.]
\end{abstract}

\title{
Plain Language Summary
}

[The MESSENGER (MErcury Surface, Space ENvironment, GEochemistry and Ranging) mission highlighted several geological terrains on Mercury's surface. Among the unexpected discoveries are small bright depressions, named hollows. Often found within impact craters, hollows have irregular shapes, flat floors and are surrounded by bright halos. Hollows are still poorly understood and their formation remains a mystery for the scientific community. Using observations made by the spectrometer on board the MESSENGER probe, we show for the first time that the spectra of hollows exhibit unique reflectance properties at near-ultraviolet to visible wavelengths, which spectrally distinguishes them from other geological terrains. Moreover, the most detailed observations show that their flat floors have different spectral characteristics from the bright halos. Our results provide new knowledge about their formation, nature and differences from other geological terrains and is consistent with them forming from sublimation processes.] 


\section{Introduction}

The MESSENGER (MErcury Surface, Space ENvironment, GEochemistry and Ranging; Solomon et al., 2007) missions highlighted the complex geological history of Mercury's crust (Denevi et al., 2009). Multi-spectral images returned by the Mercury Dual Imaging System (MDIS, Hawkins et al., 2007) and spectral observations obtained with the Mercury Atmospheric and Surface Composition Spectrometer (MASCS, McClintock \& Lankton, 2007) show that Mercury's surface has steeper spectral slopes and lower reflectance than the Moon (Robinson et al., 2008; McClintock et al., 2008; Denevi et al., 2009). Mercury's reflectance spectra exhibit a red spectral slope (i.e the reflectance increases with increasing wavelength) without silicates absorption features (Robinson et al., 2008; Izenberg et al., 2014). Although the spectral signatures are few, the surface of Mercury is spectrally and morphologically diverse. Based on spectral variation and morphology, two main terrain types and several smaller units have been identified at the surface of Mercury (Robinson et al., 2008; Denevi et al., 2009; Izenberg et al., 2014).

Low Reflectance Materials (LRM) covers approximately $15 \%$ of the planet's surface. They are among the darkest features on Mercury with an absolute reflectance 30 $\%$ below the average surface (Robinson et al., 2008). LRM have a shallower spectral slope than Mercury's mean spectrum. They have diffuse margins and are generally associated with impact structures, suggesting that this material has been excavated by impact cratering. The smooth plains represent $27 \%$ of Mercury's surface (Denevi et al., 2013). They exhibit a large range of spectral properties: from high-reflectance red plains to low-reflectance "blue" plains (blue corresponding to a less steep spectral slope than the average surface, but still with an increasing reflectance with increasing wavelength) (Denevi et al., 2009, 2013). Smooth plains on Mercury could result from effusive volcanism, impact melting or basin ejecta (Denevi et al., 2009, 2013). The majority of them share the spectral properties of the Northern Smooth Plains (NSP), the more largest (occupying 7\% of the Mercury's surface) and continuous area of smooth plains on Mercury (Denevi et al., 2013; Byrne et al., 2018).

Besides LRM and smooth plains, which are considered as large units, there are also small units that can be darkest features or high reflectance material. Dark spots are subcategories of LRM (Xiao et al., 2013), with diffuse edges but covering smaller areas (less than $100 \mathrm{~km}^{2}$ by dark spot) than LRM (often larger than $10^{6} \mathrm{~km}^{2}$ ). They have spectral slopes similar to the LRM but a lower reflectance (Xiao et al., 2013). Another spectral units are faculae which appear brighter and redder than the Mercury's average surface. Most of faculae are accepted to be pyroclastic deposits emplaced by explosive eruption. Brighter than faculae, small depressions named hollows have a reflectance approximately twice the average reflectance of Mercury (Blewett et al., 2013). Hollows-like formations have never been observed on other airless silicate bodies, which makes them an interesting case study.

Hollows are irregularly shaped, shallow, with flat floors. Their edges are usually diffused and brighter. They most often form near or directly within impact craters (Blewett et al., 2011, 2013). Some appear on crater rims, others around central peaks/rings and sometimes even in ejecta (Blewett et al., 2011, 2013). They have been often associated with LRM, Low-reflectance Blue Plains (LBP) or dark spots (Blewett et al., 2013; Xiao et al., 2013; Thomas et al., 2014a). The formation mechanism of hollows is widely discussed in literature (Blewett et al., 2013, 2018), and the loss of a volatile component is the most commonly proposed process. The arguments favoring volatile loss are i) the important abundance of volatile species in Mercury's crust as measured by X-Ray spectroscopy (Nittler et al., 2011), and ii) the preferable distribution of hollows on Sun-facing-slopes (Blewett et al., 2011, 2013; Thomas et al., 2014a). Blewett et al. (2013) proposed a formation scenario based on a vertical growth by loss of a volatile compound to a certain depth, and then an extension of the hollows by scarp-retreat. 
Due to the high abundance of sulfur in the sub-surface of Mercury (Nittler et al., 2011), CaS and MgS are commonly proposed as the volatile species (Blewett et al., 2011, 2013; Vilas et al., 2016; Lucchetti et al., 2018). This hypothesis is supported by the high concentration of exospheric calcium (Bennett et al., 2016) above the Tyagaraja impact crater in which there is a large field of hollows (Blewett et al., 2011). Vilas et al. (2016) studied in detail the Dominici and Hopper crater hollows and showed a possible absorption band centered around $630 \mathrm{~nm}$ in MDIS multispectral observations. This feature was also found in hollows from Canova and Velazquez impact craters (Lucchetti et al., 2018) and Raditladi Basin (Thomas et al., 2016). The absorption feature is often attributed to sulfides-like CaS and/or MgS (Vilas et al., 2016) or a mixture of sulfides and pyroxenes (Lucchetti et al., 2018). Thomas et al. (2016) compared hollows spectral properties with material in which they form, using MDIS multi-spectral images and the visible spectra of the Mercury Atmospheric and Surface Composition Spectrometer (MASCS). Hollows parent material seems to have lost a component that is inherently has, or a process that produced, a red spectral slope in the visible. This result is consistent with the presence of sulfides like $\mathrm{CaS}$ or $\mathrm{MgS}$ as a volatile component (Helbert et al., 2013).

Because of their possible association with volatile species, hollows allow a better understanding of the planet's geochemical evolution. The most often used instrument for spectral analysis of hollows is MDIS, due to its higher spatial resolution than that of the MASCS spectrometer. MASCS footprint sizes are often larger than the dimensions of hollows. However, MASCS has a better spectral resolution and wider spectral range than MDIS. MDIS-Wide Angle Camera (WAC) is a multispectral camera with 11 usable filters centered between 395 and $1040 \mathrm{~nm}$ and with an average spectral resolution around $60 \mathrm{~nm}$ (Hawkins et al., 2007), while MASCS spectrometer operates between 300 and $1450 \mathrm{~nm}$ with a spectral resolution around $5 \mathrm{~nm}$ (McClintock \& Lankton, 2007). The 5 hollow groups studied with more than 8 MDIS channels are those located in the Hopper, Dominici (Vilas et al., 2016; Lucchetti et al., 2018), Canova, Velazquez (Lucchetti et al., 2018) and Raditladi (Thomas et al., 2016) impact craters. Thomas et al. (2016) studied 9 additional groups using only 2 MDIS channels at about 433 and $749 \mathrm{~nm}$. Some studies (Thomas et al., 2016; Izenberg et al., 2015) used the visible part of the MASCS spectra in the hollows of the Eminescu, Raditladi, Bacho and Tyagaraja impact craters, but the spatial resolution of the observations used was not always sufficient to resolve the hollows. The comparison between the hollows and the surrounding terrain, mainly LRM (Thomas et al., 2016) and Facula (Izenberg et al., 2015), were made using reflectance ratios of 2 channels of MASCS or MDIS.

In this work, we used MASCS spectra at the best spatial resolution available (less than $0.5 \mathrm{~km}$ /footprint) to improve the spectral resolution and the wavelength range compared to previous studies of hollows (Vilas et al., 2016; Thomas et al., 2016; Lucchetti et al., 2018). We found 4 large fields of hollows spatially resolved with MASCS observations located in the Tyagaraja, Hopper, Warhol and Eminescu impact craters. The main objective of this study is to improve our understanding of the spectral characteristics of hollows. In particular, we will characterize the hollows in the near-ultraviolet through near-infrared, using parameters derived from full spectral coverage. In addition, we will examine the MASCS spectra for absorptions such as those observed in MDIS filters (Vilas et al., 2016; Lucchetti et al., 2018), directly comparing the MASCS and MDIS observations in the Hopper crater.

\section{Datasets and Method}

The data used in this work are obtained from the final delivery of products at the Planetary Data System (PDS) and have the best calibration (photometric and radiometric) performed by the MESSENGER team (Izenberg \& Holsclaw, 2017). 


\subsection{Selection and correction of the spectra}

MASCS Visible and InfraRed Spectrometer (VIRS) is composed of two detectors: a visible (VIS) detector operating between 300 and $1050 \mathrm{~nm}$ and a near-infrared (NIR) detector sensitive to wavelengths between 900 and $1450 \mathrm{~nm}$. The NIR detector is more dependent on the orbital, seasonal and instrumental temperature variations (Izenberg et al., 2014). Consequently, the signal-to-noise ratio varies substantially between VIS and NIR detector, which complicates analysis (Izenberg et al., 2014).

Therefore, we selected the spectra measured in the two lowest temperature regimes of the instrument $\left(\leq 25^{\circ} \mathrm{C}\right)$, except for 2 orbits located in Tyagaraja (see section 2.3) where low temperature data are not available. We selected these ranges of temperature because the reference spectrum (Izenberg et al., 2014) used in this analysis (see section 2.2 ) is based on spectra obtained at temperatures lower than $30^{\circ} \mathrm{C}$. Also, we selected a grating temperature under $40^{\circ} \mathrm{C}$, because if we selected less than $25^{\circ} \mathrm{C}, 78 \%$ of data corresponding to the hollows would be removed.

Additional processing is applied to the data using the method developed by Besse et al. (2015). This approach, based on 3 main steps (as described below) allows the merger of the VIS and NIR data in a combined spectrum. The first step consists to remove the outliers deviating by more than 2 sigma from the mean. Only the most distant outliers are removed using this method, which represents less than $1 \%$ of the measurements by the VIS detector and less than $4 \%$ in the NIR. In a second step, a moving average window of three points is applied to the entire VIS and NIR to smooth the data. Smoothing implies a loss of information on the signal without consequences on the analysis performed in this study. Finally, an offset is applied to the NIR to combine the two parts of the spectrum (Besse et al., 2015). The approach tested on lunar spectra by Besse et al. (2015), has been validated for observations of Mercury's surface. Moreover, this method allows the scatter of the channel-to-channel reflectance to be reduced, especially in the NIR domain. Besse et al. (2015) and Besse et al. (2020) demonstrated that the NIR channel could be analyzed with reasonable confidence in the study of pyroclastic deposits.

\subsection{Spectral analysis}

Our analysis is focused on spectral parameters encompassing the entire spectral range of MASCS from the near-ultraviolet to the near-infrared.

- In the near-ultraviolet (UV), the UV-downturn described by Goudge et al. (2014), which characterizes the drop of the reflectance shortward of $350 \mathrm{~nm}$, is calculated. This parameter gives the offset between measured reflectance and the expected reflectance at 3 wavelengths $(300,325$ and $350 \mathrm{~nm})$ if the reflectance had the same slope in the UV and in the visible. The mathematical definition of the UV-Downturn is (Goudge et al., 2014):

$$
U V-\text { downturn }=\text { Depth }_{300}+\text { Depth }_{325}+\text { Dept }_{350}
$$

where,

$$
\begin{aligned}
& \text { Depth }_{300}=\left(R_{(401)}-(401-303) S_{V I S}\right) / R_{(303)} \\
& \text { Depth }_{325}=\left(R_{(401)}-(401-324) S_{V I S}\right) / R_{(324)} \\
& \text { Depth }_{350}=\left(R_{(401)}-(401-350) S_{V I S}\right) / R_{(350)}
\end{aligned}
$$

and,

$$
S_{V I S}=\frac{R_{(750)}-R_{(445)}}{750-445}
$$

where $R_{(\lambda)}$ is the reflectance at the wavelength $\lambda$ given in nanometers.

- In the visible, the absolute reflectance at $750 \mathrm{~nm}$ (R750), which is an average value of three points due to the smoothing of the data (see section 2.1), is used. 
- For the characterization of the spectral slope, we calculate the visible (VIS) slope as defined in Besse et al. (2015) between 445 and $750 \mathrm{~nm}$. We extended the range of wavelength to the NIR domain $(1400 \mathrm{~nm})$ to define the visible to near infrared (VISNIR) slope. The slopes are determined by a linear fit:

$$
\text { Reflectance }=a \lambda+b \quad(\mathrm{a}=\text { slope })
$$

where $445 \mathrm{~nm} \leq \lambda \leq 750 \mathrm{~nm}$ for the VIS-slope and $445 \mathrm{~nm} \leq \lambda \leq 1400 \mathrm{~nm}$ for the VISNIR-slope.

- After a visual inspection of the spectra, a parameter named Curvature was defined. The Curvature is calculated before $600 \mathrm{~nm}$, that is the point at which the spectrum changes from UV curvature to near-infrared slope (arbitrarily by visible inspection). This parameter is represented by the coefficient of the squared power of the polynomial fit (degree 2) of the spectrum between 300 and $600 \mathrm{~nm}$ :

$$
\text { Reflectance }=c \lambda^{2}+a \lambda+b \quad(\mathrm{c}=\text { curvature })
$$

Random examples are shown in Figure 1.

The slopes and the Curvature parameter are normalized to the reference spectrum provided by Izenberg et al. (2014) who computed it from the average of 850,000 spectra of Mercury's surface. By definition, slopes and Curvature are equal to 1 for the average surface of Mercury. The UV-downturn parameter previously estimated to 3.0 for Mercury's background, using Izenberg et al. (2014) reference spectra, was re-evaluated as 3.1 by Besse et al. (2020). 


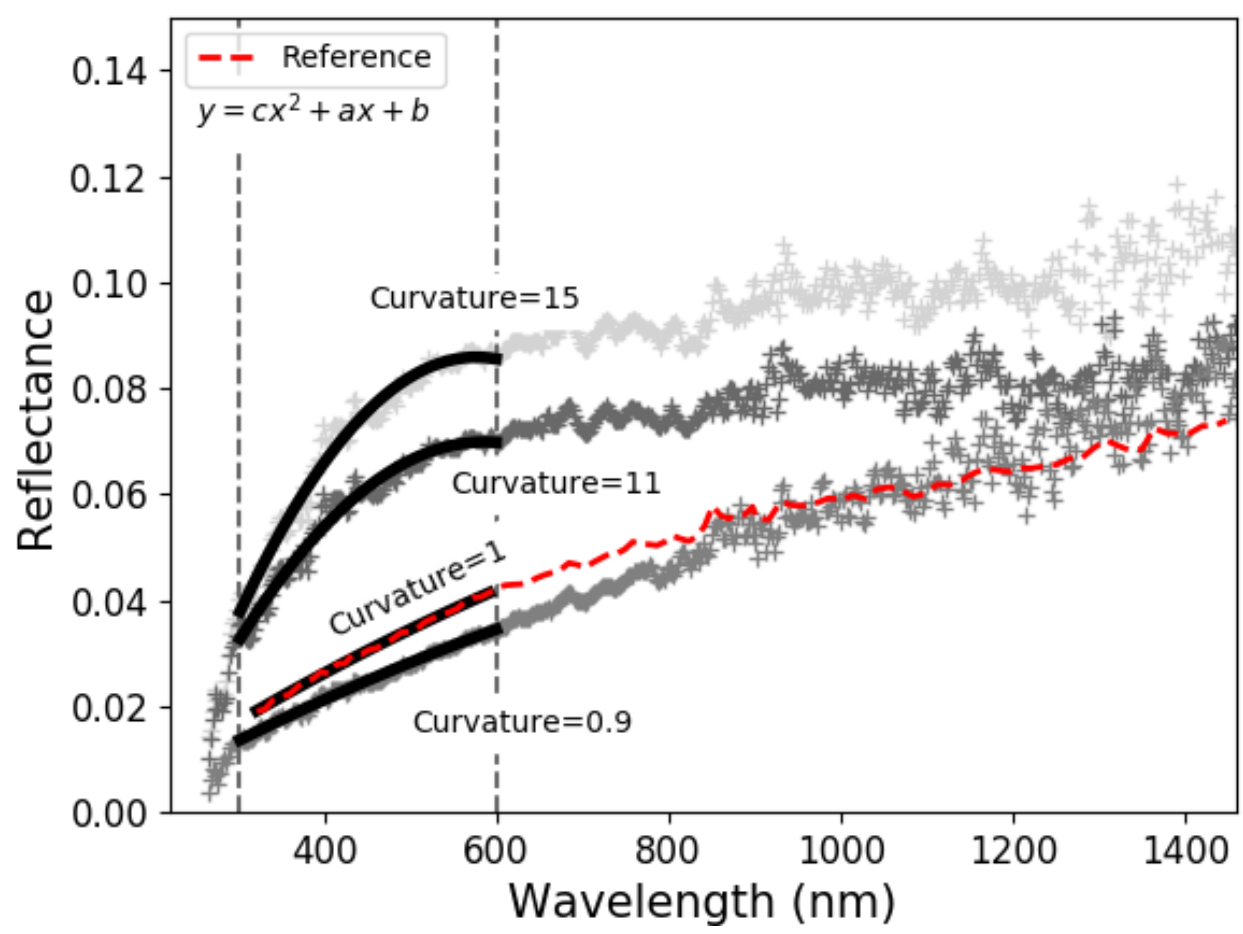

Figure 1. Example of calculated Curvature on MASCS spectra, and on Mercury's reference spectrum (Izenberg et al., 2014). The black curve represents the polynomial fit (degree 2) of the spectrum between 300 and $600 \mathrm{~nm}$. The Curvature parameter corresponds to the variable $\mathrm{c}$ in the equation of the polynomial curve. Furthermore, the Curvature coefficient is normalized to the reference spectra (red, Izenberg et al., 2014) such that the Curvature of the reference spectrum is equal to 1 . The spectra with the highest reflectance were randomly selected in Eminescu's hollows (ob4_14327_010045 and ob4_14326_164731, see section 2.3). The spectrum with the lowest reflectance was randomly selected in the Northern smooth plains (ob2_12187_063041, see section 2.4).

\subsection{Hollows observations with MASCS}

MDIS instrument acquired a global and uniform coverage in 8 of its 11 colour filters of more than $90 \%$ of the planetary surface during the orbital phase (Domingue et al., 2017). On the other hand, the MASCS instrument is a point spectrometer and thus only observed discrete areas. Dimensions of MASCS footprints vary between $0.1 \times 3 \mathrm{~km}$ to over $6 \mathrm{x} 7 \mathrm{~km}$ (Izenberg et al., 2014). Hollows are small depressions, which range in size between several tens of meters to several kilometers (Blewett et al., 2018), hence, they are less likely to be observed with MASCS.

Using the list defined by Thomas et al. (2014a), we found four hollows observed with sufficient spatial resolution by MASCS. They span a large range of longitudes, but a restricted range of latitudes $\left(15^{\circ} \mathrm{N}\right.$ to $\left.15^{\circ} \mathrm{S}\right)$. Observable hollows are located in the Tyagaraja, Hopper, Eminescu, and Warhol impact crater floors, as shown in Figure 2.

High-resolution images produced by the Narrow Angle Camera (NAC) of the MDIS instrument are used for selecting MASCS footprints. Footprints are represented by an 

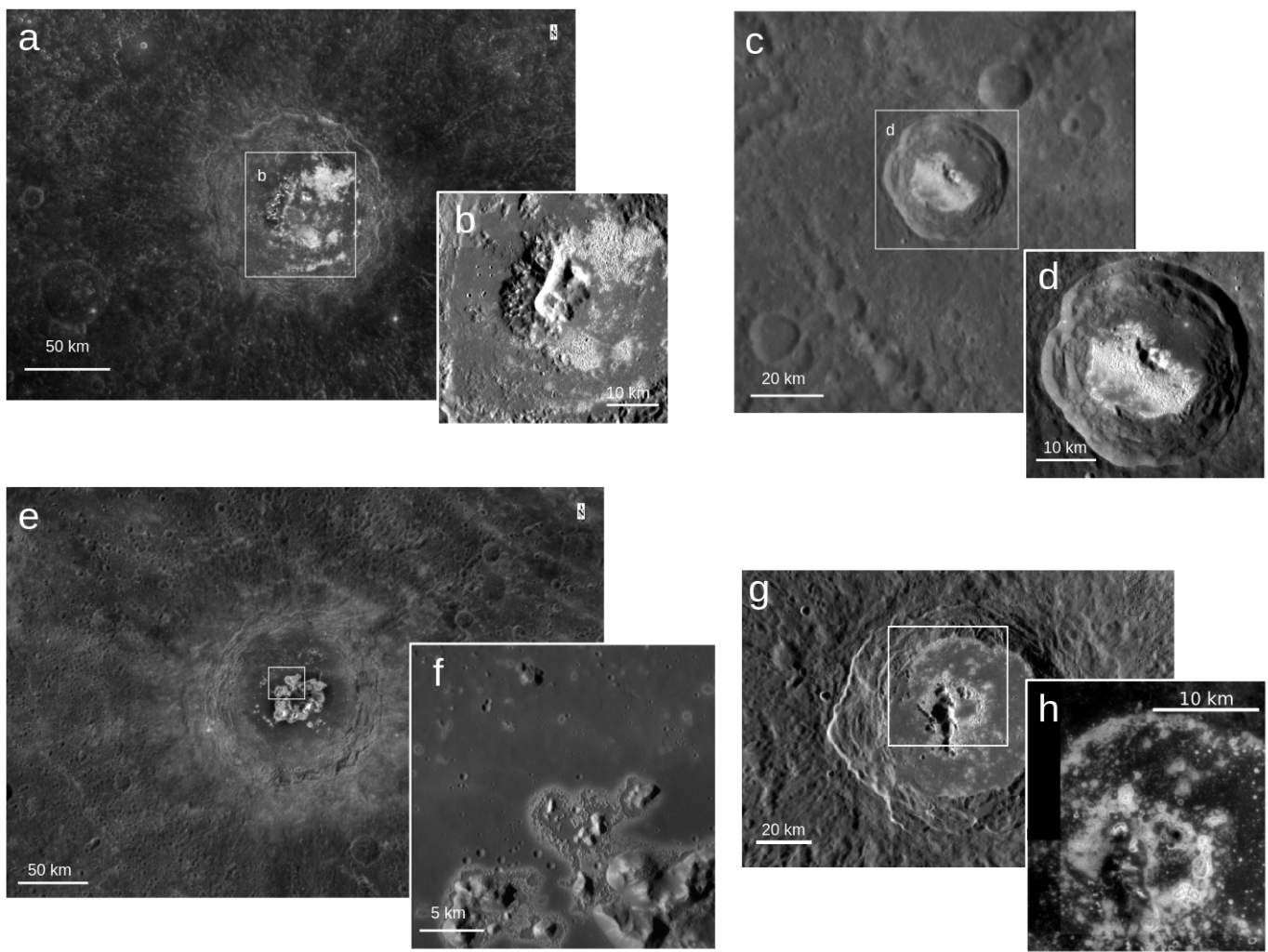

Figure 2. Hollows analyzed in this work. a) Tyagaraja impact crater $\left(3.89^{\circ} \mathrm{N}, 211.10^{\circ} \mathrm{W}\right.$; from Wide Angle Camera (WAC)-MDIS image EW1009232948G, 383 m/pixel). b) Zoom on the north-east hollows in Tyagaraja crater (from Narrow Angle Camera (NAC)-MDIS image EN0242713071M, $130 \mathrm{~m} /$ pixel). c) Hopper impact crater $\left(12.44^{\circ} \mathrm{S}, 304.40^{\circ} \mathrm{W}\right.$; EN1048482439M, $110 \mathrm{~m} /$ pixel). d) Zoom on hollows in Hopper crater (EN0223616383M, $49 \mathrm{~m} /$ pixel). e) Eminescu impact crater $\left(10.66^{\circ} \mathrm{N}, 245.79^{\circ} \mathrm{W}\right.$; EW0234069376G, $476 \mathrm{~m} /$ pixel). f) North-north-east part of the central peak in Eminescu impact crater (EN0251632156M, 35 m/pixel). g) Warhol impact crater $\left(2.55^{\circ} \mathrm{S}, 6.27^{\circ} \mathrm{W}\right.$; MDIS_RTM_N01_009974_5942839_1, $\left.92 \mathrm{~m} / \mathrm{pixel}\right)$. h) North part of Warhol impact crater (EN1034982894M and EN1035068908M, 56 m/pixel).

ellipse on the NAC images and are selected manually. Figure 3 shows footprints used in this study. For each site, at least one footprint located in the host impact crater floor is selected. The spatial resolution of MASCS in the Tyagaraja, Hopper and Warhol impact craters allows the analysis of the properties of the hollows group (Figure 3). In the case of Eminescu, a particular hollow can be analyzed. Moreover, the footprint size in Eminescu is sufficient to distinguish the hollow floor from the surrounding halo.

As for the Hopper impact crater, data of two orbits are used. For the largest footprints, the spatial resolution of MASCS is too large (around $10 \mathrm{~km} /$ footprint) to include only material from the hollows (Figure 3). Consequently, the spectra obtained from these footprints are thought to be representative of a mixing between hollows and crater floor material. By superimposing each footprint on the NAC image, we are able to estimate the proportion of hollows field contained in the ellipse with uncertainty of $+/-5 \%$. 

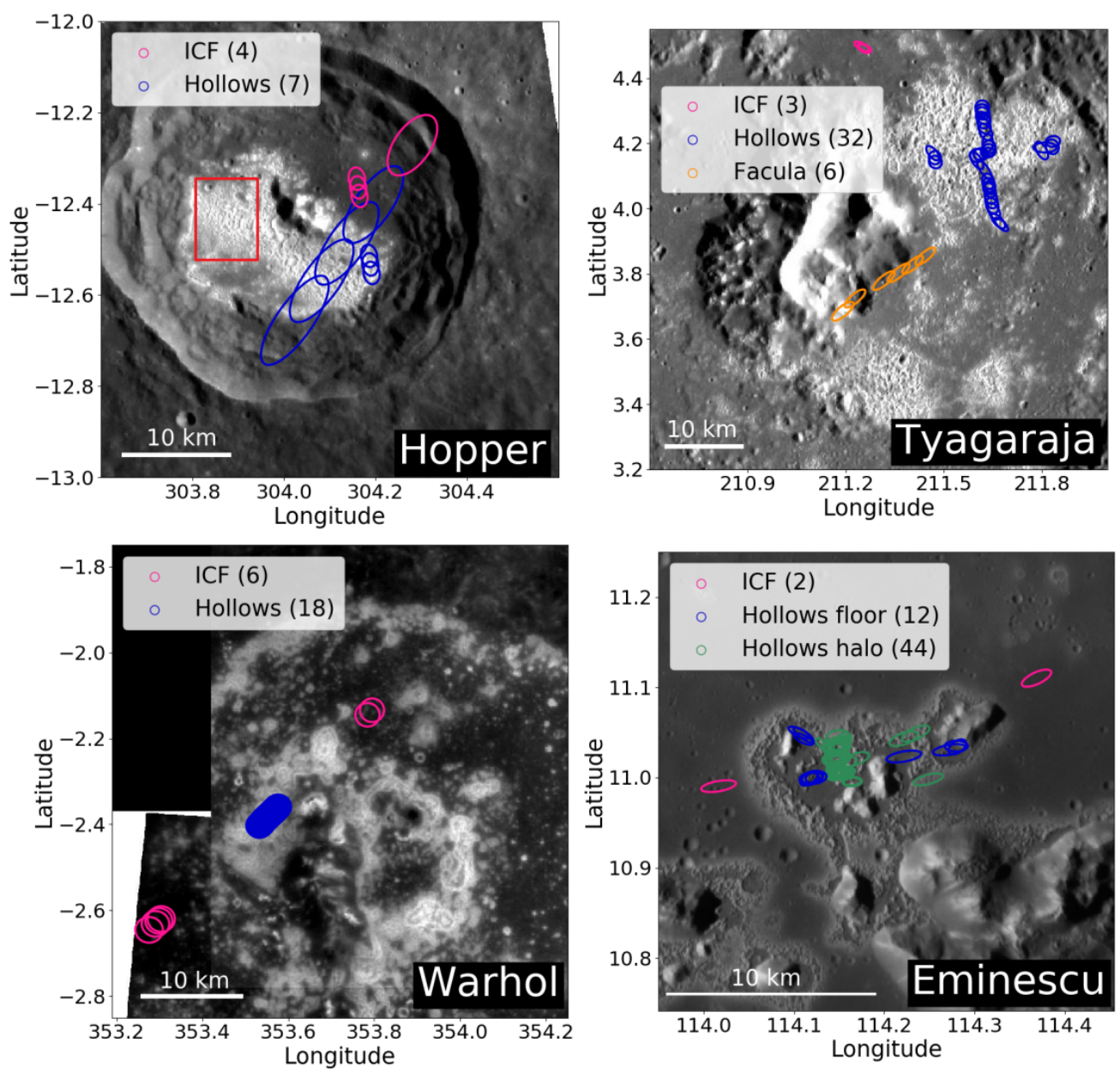

Figure 3. Representation of MASCS footprints used in this study. a) Hopper impact crater floor (ICF) hollows and footprints from 2 orbits (ob2_12268_133540, ob3_13276_093728) with a spatial resolution between 0.6 to $10 \mathrm{~km} /$ footprint. The red rectangle shows the area sampled by Vilas et al. (2016). b) Tyagaraja hollow's field, the first orbit (ob4_14128_101058, 0.7 to $2 \mathrm{~km}$ /footprint) is used for hollows and impact crater floor (ICF) and the second one (orange: orb_11346_182504, 2.5 to $3.6 \mathrm{~km} /$ footprint) is used for Tyagaraja facula. c) Warhol impact crater, the orbit used is ob4_15064_011135 (around $2.5 \mathrm{~km} /$ footprint). d) Eminescu impact crater central peak (north-west part), the two orbits used are very similar (ob4_14327_010045, ob4_14326_164731) and are obtained one day apart. The spatial resolution varies between 0.3 to $1.7 \mathrm{~km} /$ footprint. The NAC images used are the same as in the previous figure. The number in the legends give the number of MASCS observations for each hollows, Impact crater floor (ICF) and facula in Tyagaraja.

\subsection{Observations of other geological units}

Hollows are among the brightest features at the surface of Mercury. In order to understand their spectral characteristics in the near-ultraviolet to near-infrared wavelength, we compare them with MASCS observations of other geological units. Several terrains around the Mercury's surface are used: Dark spots (DS), Low reflectance material (LRM), faculae and Northern Smooth Plains (NSP). 


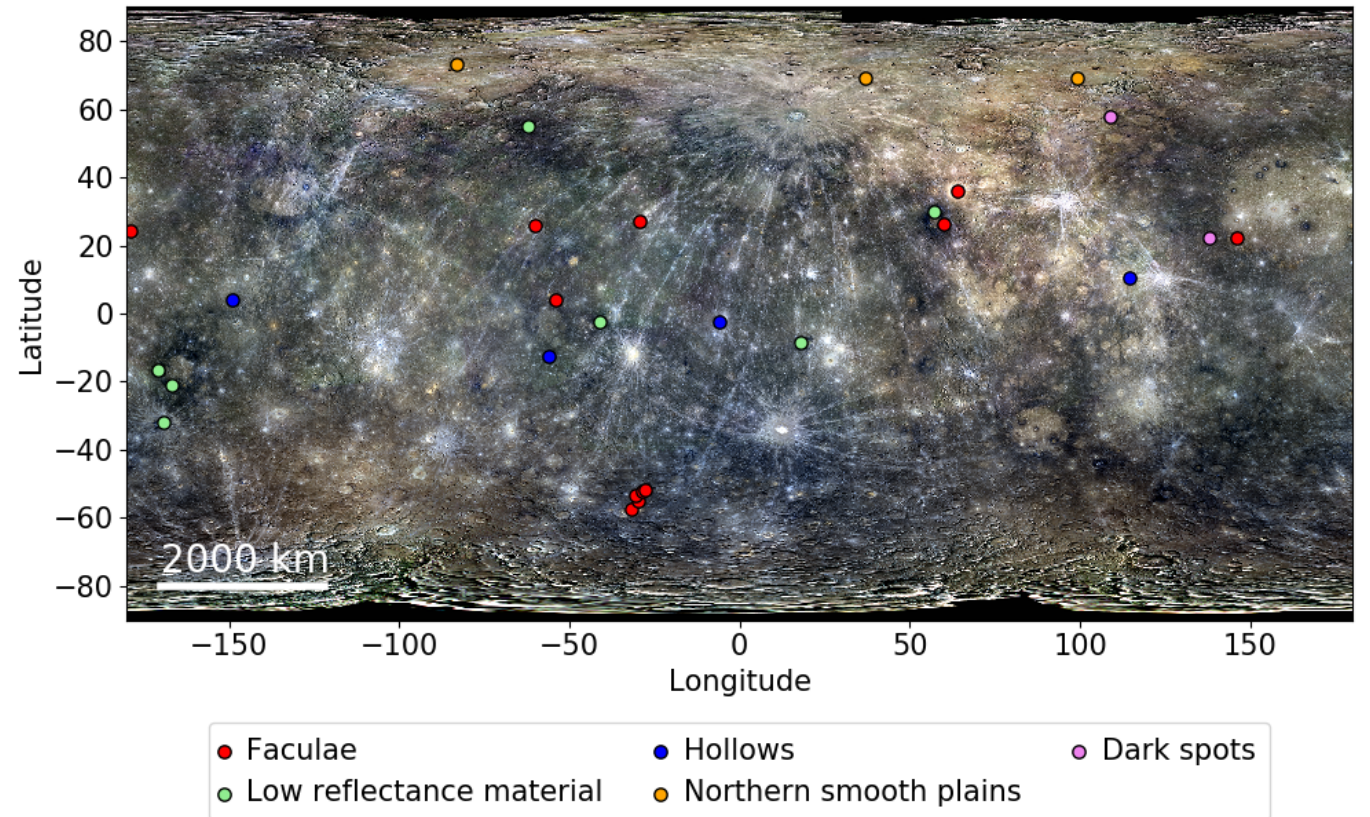

Figure 4. Global distribution of hollows (blue dots) analyzed in this work, relative to other geological units. Low reflectance material, northern smooth plains and faculae areas selected are similar to the size of the dots. The background is a MDIS color global mosaic (3-filters) red: 1000 nm, green: $750 \mathrm{~nm}$, and blue: $430 \mathrm{~nm}$ (665 m/pixel; Denevi et al., 2018).

Hollows are often found nearby faculae, as in the Tyagaraja impact crater (Blewett et al., 2011). The volatile species causing volcanic eruptions could be a source of volatile material for the formation of hollows as well (Blewett et al., 2011, 2013; Thomas et al., 2014a). To investigate their possible relationship, we compare the spectral properties of hollows and faculae. We selected MASCS observations in the facula of the Tyagaraja impact crater (Figure 3). Moreover, spectral properties of faculae were studied by Goudge et al. (2014) and Besse et al. (2015) using the MASCS spectrometer. Besse et al. (2020) determined the diameter of 14 faculae, and 12 of them (Figure 4 and Table S3) have instrumental conditions (i.e. temperatures, see section 2.1) similar to those determined suitable in this analysis, and are thus used for comparison to hollows.

Northern smooth plains (NSP) occupy more than 7\% of Mercury's surface (Byrne et al., 2018) and are, similarly to faculae, a consequence of volcanic activity. We have selected 3 regions with a large number of MASCS footprints passing through NSP regions (Figure 4 and Table S4).

Table 1 lists the number of observations used in each unit and illustrates the difficulty in finding MASCS footprints in smaller units like hollows and DS. Although we selected regions in LRM and NSP with the least surface feature heterogeneity (impact 
craters, ejecta, rays, possible hollows and dark spots that can modify spectral properties), we eliminated spectra which deviate more than 3 sigma from the median.

Table 1. Number of MASCS observations selected per geological unit.

\begin{tabular}{lll} 
Geological unit & Description & $\begin{array}{l}\text { Number of } \\
\text { observations }\end{array}$ \\
\hline $\begin{array}{l}\text { Low Reflectance } \\
\text { Material }\end{array}$ & $\begin{array}{l}\text { Dark material excavated by impact cratering and poten- } \\
\text { tially parent material of hollows (Thomas et al., 2016). }\end{array}$ & 1115 \\
\hline $\begin{array}{l}\text { Northern } \\
\text { Smooth Plains }\end{array}$ & $\begin{array}{l}\text { Largest continuous plains resulting of effusive volcanism } \\
\text { (Denevi et al., 2013; Byrne et al., 2018). }\end{array}$ & 1352 \\
\hline Faculae & $\begin{array}{l}\text { Pyroclastic deposits formed through explosive volcanic } \\
\text { processes. (Kerber et al., 2009, 2011) }\end{array}$ & \\
\hline Dark spots & $\begin{array}{l}\text { Sub-category of LRM at smaller scale. Hollows commonly } \\
\text { occupy the centers of the observed dark spots (Xiao et al., }\end{array}$ & \\
& 2013). & \\
\hline Hollows (4) & $\begin{array}{l}\text { Irregularly shaped depressions, shallow, with flat floor } \\
\text { and surrounded by bright halo (Blewett et al., 2018). }\end{array}$ \\
\hline
\end{tabular}




\section{Spectral properties of hollows from near-ultraviolet to near-infrared}

Before starting the analysis with the spectral parameters, the average spectrum of each geological unit is derived from the corrected spectra (see section 2.1). The mean spectrum of hollows differs from others by its unique shape in the near-ultraviolet and a higher reflectance while the others average spectra overlap across the entire spectral range of MASCS (Figure 5).

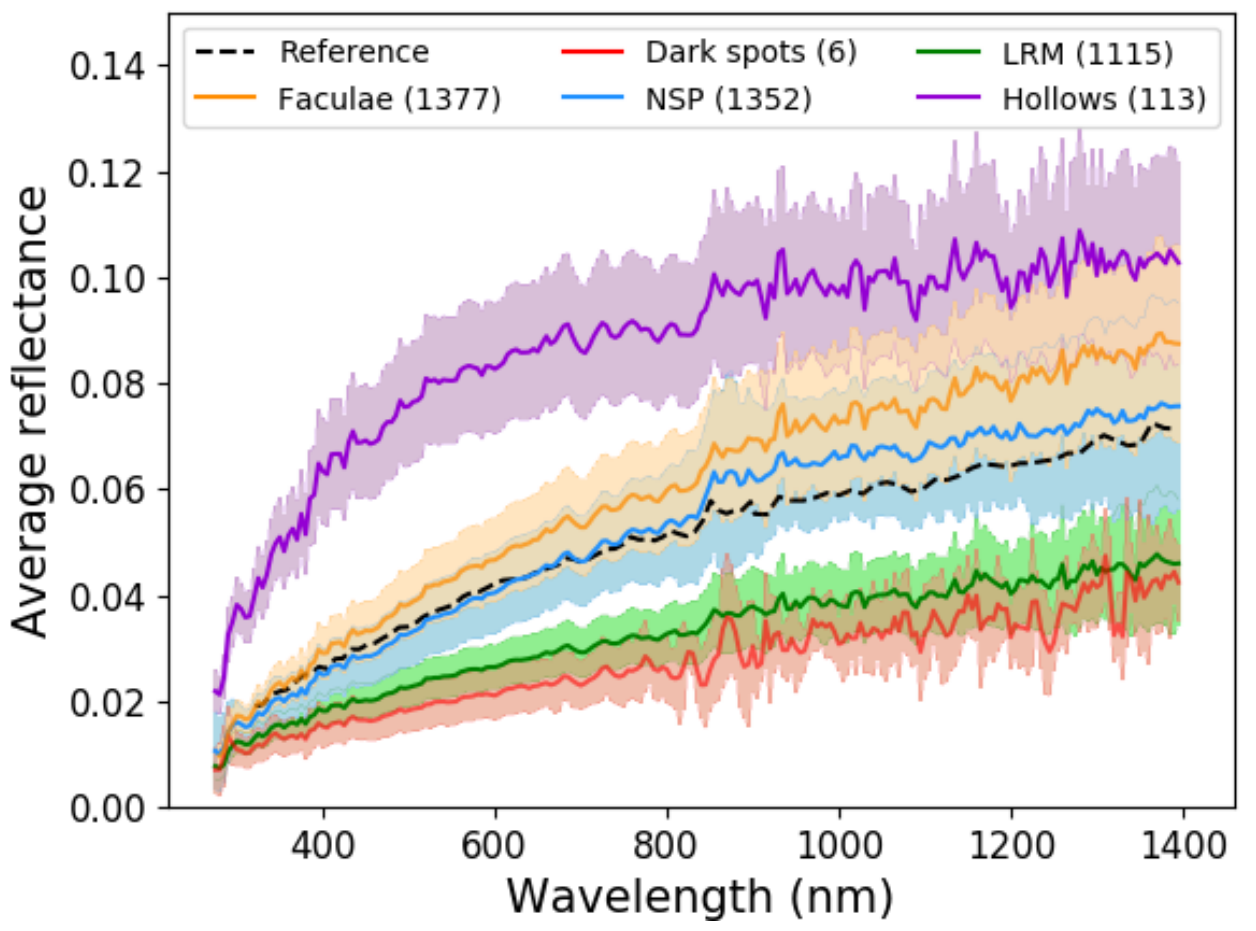

Figure 5. Average reflectance spectrum of each geological unit described in section 2.3 and 2.4. LRM: Low Reflectance Material, NSP: Northern Smooth Plains. The shaded regions are error bars, which correspond to the standard deviation of the spectra around the mean. Reference is the average spectrum of Mercury's surface (Izenberg et al., 2014). 


\subsection{Visible to near-infrared properties}

The MASCS reflectance at $750 \mathrm{~nm}$ is approximately twice as high for the hollows than Mercury's mean spectrum (Figure 5 and 6 ). While the faculae are also bright features on the surface of Mercury (Kerber et al., 2009), they have a significant lower reflectance than the hollows at all wavelengths (Figure 5). These results are consistent with those obtained from MDIS observations, which show that the hollows are among the brightest geological units on the surface of Mercury (Blewett et al., 2011, 2013).

All geological features observed with MASCS in this study have positive spectral slopes (reflectance increases toward longer wavelengths). VIS-slope of hollows ranges from 0.38 to 1.45 (Figure 6 ).

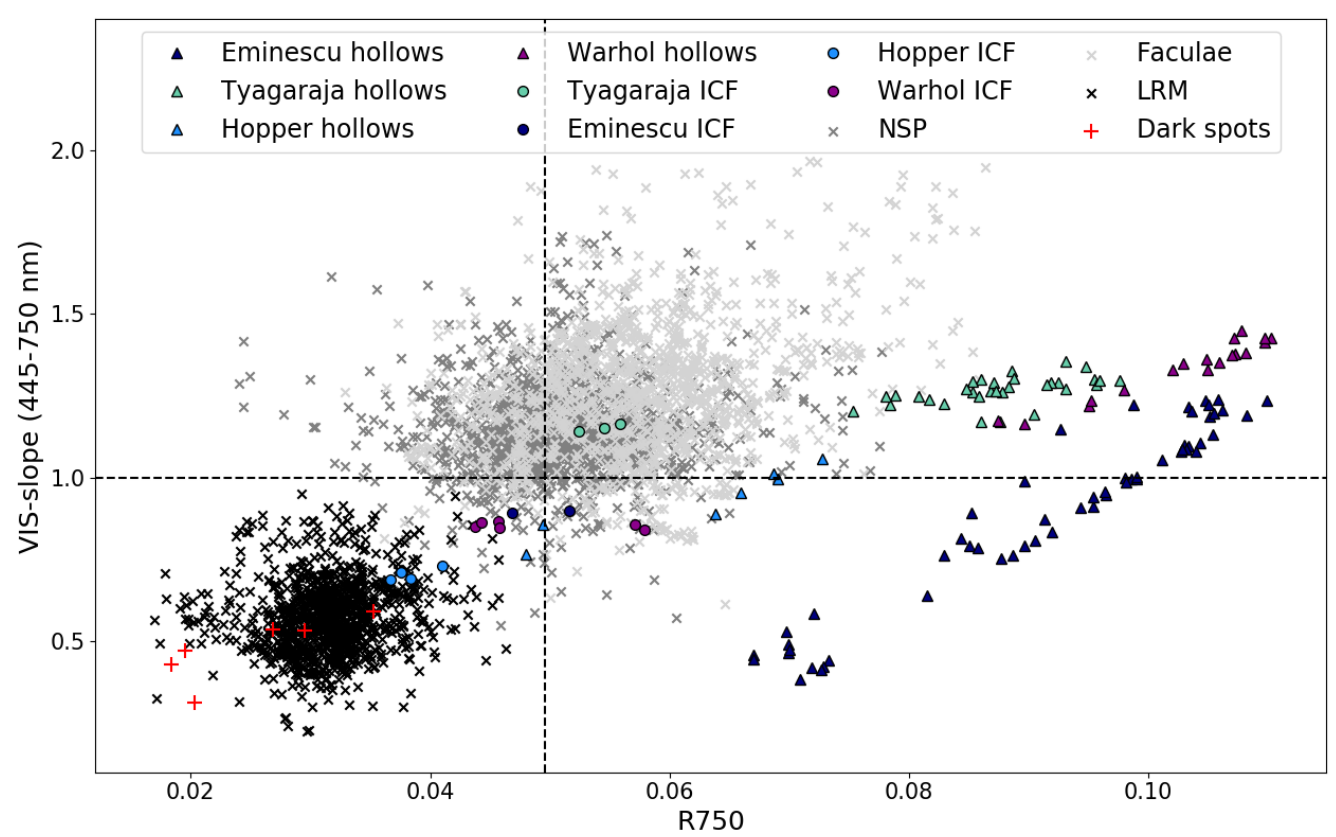

Figure 6. VIS-slope parameter versus R750 parameter in hollows and in various geological units: Low Reflectance Material (LRM), faculae, Northern Smooth Plains (NSP), dark spots and Impact Crater Floor (ICF). Black dashed lines show reflectance at $750 \mathrm{~nm}$ and value of the VIS-slope parameter for the average surface of Mercury. In general, the hollows are brighter than other units on Mercury.

MDIS spectral analysis showed that the hollows are less red than the Mercury's mean surface (Blewett et al., 2013; Thomas et al., 2016), i.e. a VIS-slope parameter lower than 1. However, those results were obtained using only two spectral channels (433 and $749 \mathrm{~nm}$ ). Thomas et al. (2016) estimated the visible slope by dividing images at $430 \mathrm{~nm}$ by images at $750 \mathrm{~nm}$ for MDIS and/or calculating the ratio of reflectance at $445 \mathrm{~nm} / 750 \mathrm{~nm}$ in MASCS data. In our study, the parameter VIS-slope is derived from the best fit line between 445 and $750 \mathrm{~nm}$, that is from around 60 data points. Therefore this parameter is more sensitive to the overall shape of the spectra compared to a ratio using 2 bands. The curvature of the hollows' mean spectrum at the shortest wavelengths (Figure 5) could explain why the VIS-slope is higher than 1 for many of the hollows.

At longer wavelengths, the hollows mean spectrum is flatter than that of the mean spectra of other geological units (Figure 5). The VISNIR-slope parameter allows the study of the effect of the near-infrared wavelengths. The hollows spectra have a VISNIR-slope 
lower than their VIS-slope (Figure 7). In fact, $76 \%$ of the normalized spectral slopes in the VIS to NIR are lower than 1, while only $30 \%$ are in the visible. For the other geological units, the difference between VIS-slope and VISNIR-slope is not as significant (Figure 6 and 7), suggesting that the hollows spectra are flatter in the NIR than the spectra of the other units.

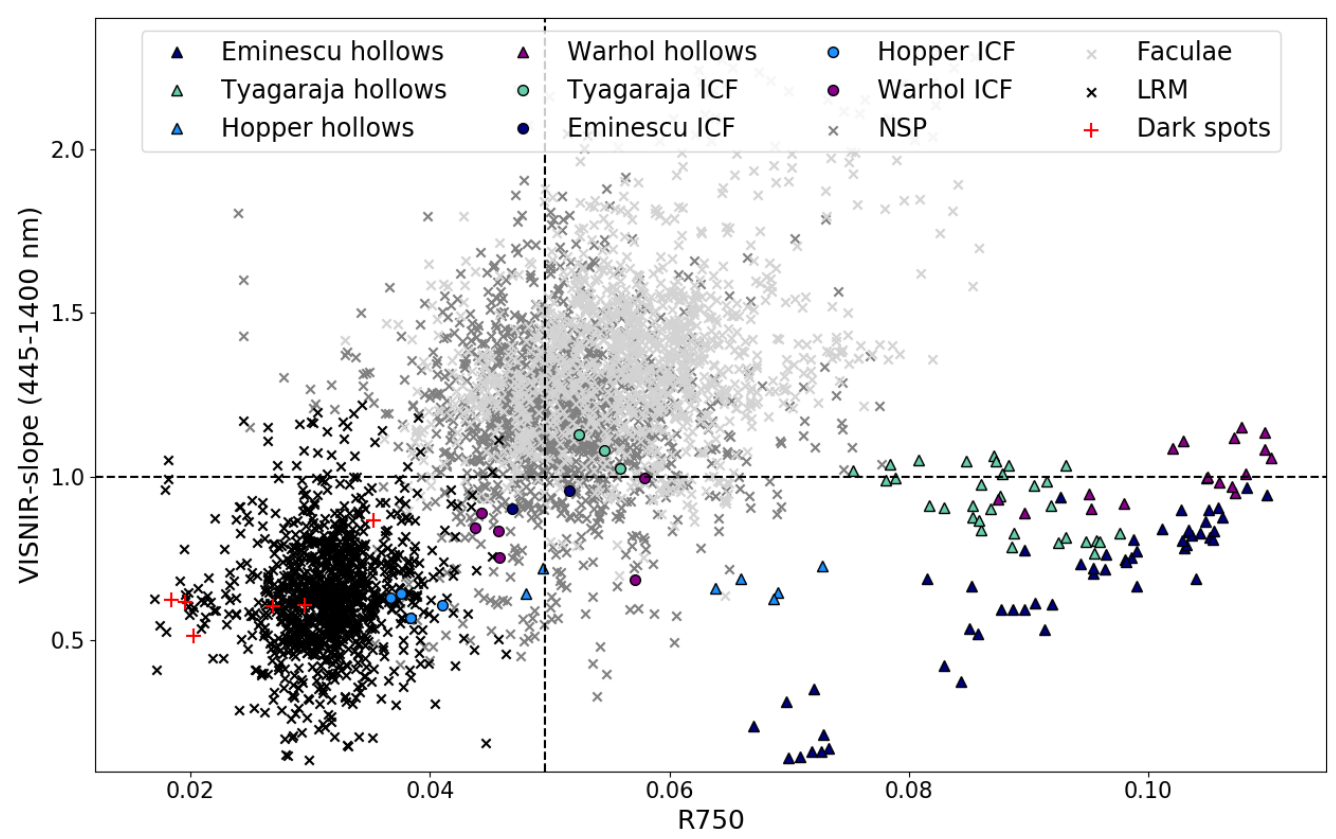

Figure 7. VISNIR-slope parameter versus R750 parameter in hollows and various geological units: Low Reflectance Material (LRM), faculae, Northern Smooth Plains (NSP), dark spots and Impact Crater Floor (ICF). Black dashed lines show reflectance at $750 \mathrm{~nm}$ and value of the VISNIR-slope parameter for the average surface of Mercury. In general, hollows have a visible to near-infrared slope less steep than the Mercury's mean spectrum (Izenberg et al., 2014).

Space weathering affects the reflectance spectra by increasing the spectral slope in the visible-near-infrared due to the presence of submicroscopic iron (smFe) (McCord \& Adams, 1972a; McCord \& Adams, 1972b; Fischer \& Pieters, 1994; Domingue et al., 2014). Hollows are thought to be among the most recent geological units on the surface of Mercury (Blewett et al., 2018). Their reduced spectral slope in the VISNIR could be a consequence of their relatively young age.

Although the spectral parameters in the visible (VIS-slope, VISNIR-slope and R750) allow assumptions to be made about the hollows, they overlap with those of other geological units. Thus at visible wavelengths, no spectral parameters used in this paper allow the hollows to be uniquely characterised. 


\subsection{Near-ultraviolet properties and spectral curvature as diagnostic of hollows}

The MASCS reflectance spectra of hollows reveal the highest UV downturn and curvature at wavelengths shortward of $600 \mathrm{~nm}$. The strength of the downturn and the curvature differs among the hollows and are linearly correlated (Pearson coefficient correlation of 0.88 with a significance level of $99.9 \%$ ). For the other geological units, no correlation was found between the two parameters. This result implies a process or material influencing the two spectral parameters in the hollows that would not be found in other geological units. Several factors could potentially influence the downturn of the reflectance in the ultraviolet, we mention three hypotheses here: 1) the space weathering, 2) the grain size of the material forming the hollows and 3) the presence of sulfides.

As mention on the previous section, space weathering increases the spectral slope in the VISNIR, resulting in a reddening of the spectrum. (Domingue et al., 2014). In the near-ultraviolet, it will start to decrease the slope (bluing), especially between 300 and $400 \mathrm{~nm}$ (Hendrix \& Vilas, 2006; Vilas \& Hendrix, 2015). This change of the slope in the near-ultraviolet has been interpreted by Hendrix and Vilas (2006) as the result of the formation of nanophase iron coatings on mineral grains. The hypothesis of space weathering could explain the slopes of the hollows spectra in the near-ultraviolet and visiblenear-infrared. Research on the effects (Hendrix \& Vilas, 2006; Vilas \& Hendrix, 2015) of space weathering on reflectance spectra does not indicate a curvature of the spectrum between 300 and $600 \mathrm{~nm}$, making the idea of space weathering, in the absence of other possible factors, less likely.

Another possible contributor to the high UV-downturn in the hollows is a difference in grain size between hollows and other geological units. Laboratory studies have shown that as the grain-size decreases, the UV-downturn increases for common planetary minerals (Cloutis et al., 2008). If the particle size is the only one contributing factor to the high downturn of the hollows, then the hollows have finer grain size than the other geological units studied here. Phase ratio analysis suggests that hollows around the central peak of Eminescu crater have finer particle sizes than the surrounding crater floor (Blewett et al., 2014). A second phase ratio analysis confirms on a sample of eight sites that hollows have finer grain size than their adjacent terrains (Thomas et al., 2016). However, hollows often have UV-downturn close to that of their host crater floor, which is particularly the case for Eminescu (Figure 8). Consequently, grain size does not appear to be the only one factor influencing this spectral parameter. In addition, no studies have shown that particle size has an impact on the curvature of the spectrum between 300 and $600 \mathrm{~nm}$, making this idea unlikely.

An alternative possibility to the specific properties of hollows in the UV is a specific composition. Sulfides are the most commonly cited candidates for the volatile species at the origin of the hollows (Blewett et al., 2011, 2013; Vilas et al., 2016; Lucchetti et al., 2018; Helbert et al., 2013). Laboratory measurements carried out under the conditions of MASCS observations show that the spectra of fresh CaS, NaS and MgS (2 samples) have a strong absorption band between 280 and $350 \mathrm{~nm}$, centered at $300 \mathrm{~nm}$ (Varatharajan et al., 2019). The Mercury daytime temperatures have different implications on the spectral features of these samples (Varatharajan et al., 2019). For one of the MgS sample, the absorption features disappear after the thermal processing at Mercury's surface temperatures. The spectral contrast of the absorption features around $300 \mathrm{~nm}$ is slightly subdued for the heated $\mathrm{CaS}$ and the second heated MgS sample while the heated NaS shows stronger UV spectral features (Varatharajan et al., 2019). In consequence, the spectral features of $\mathrm{CaS}, \mathrm{MgS}$ and $\mathrm{NaS}$, even under the temperature conditions of Mercury, may involve the high UV downturn of the hollows. The curvature of laboratory spectra of Mercury analogs has not been studied, however it would be interesting to compare the Curvature of spectra measured in the laboratory on materials possibly representative of hollows with our values in order to determine if this curvature can be related to the pres- 
ence of sulfides or any other minerals. For example, we calculated the reflectance curvature for one of the MgS sample presented by Varatharajan et al. (2019) and obtained a value of 22 , slightly higher than our hollow values.

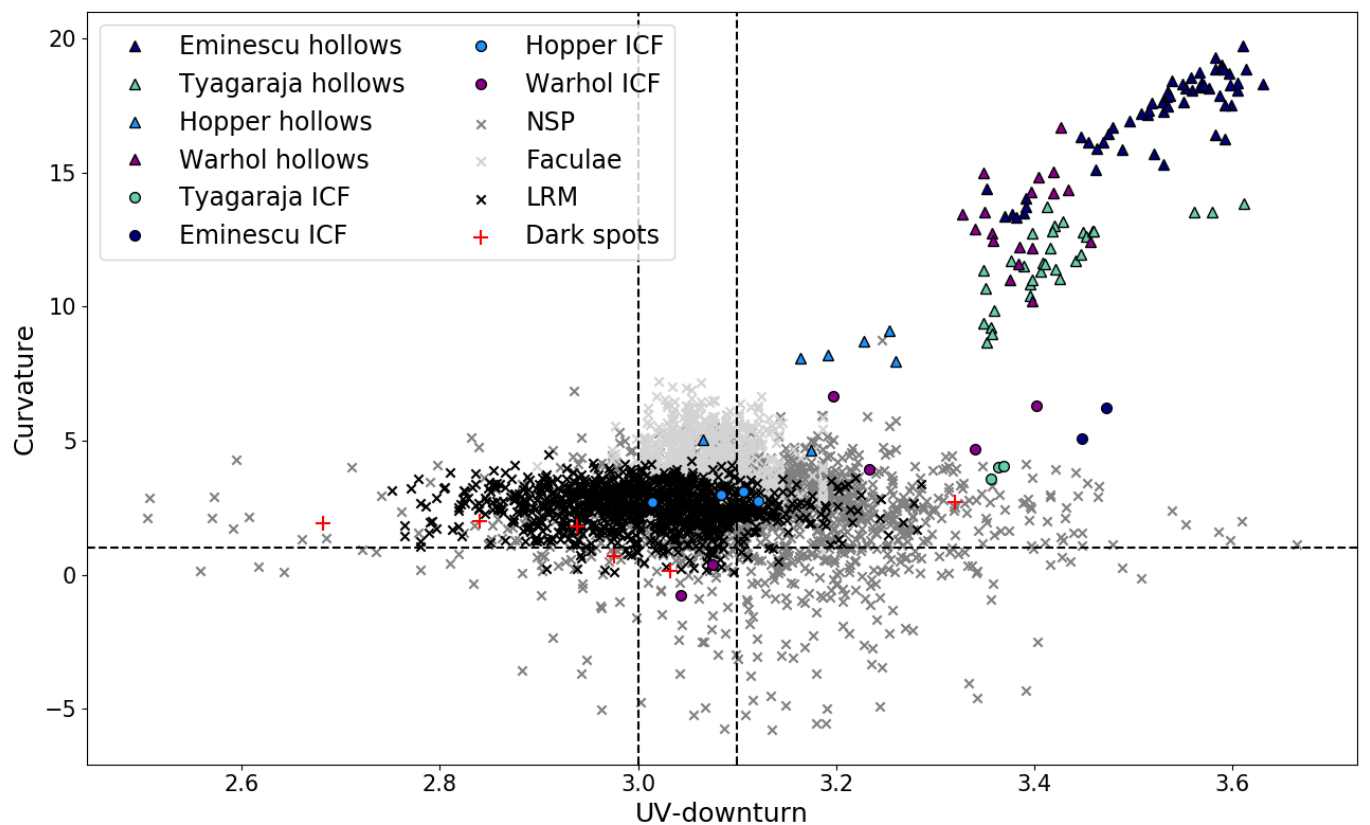

Figure 8. Curvature versus UV-downturn for hollows and the various geological units: Low Reflectance Material (LRM), faculae, Northern Smooth Plains (NSP), dark spots and Impact Crater Floor (ICF). Black dashed lines show the values of these parameters for the mean spectrum of Mercury. The UV-downturn estimated at 3.0 for the average surface was re-evaluated at 3.1 (Besse et al., 2020). The hollows stand out slightly by UV-downturn parameter and largely by Curvature parameter. 


\subsection{Influence of abundance: The Case of Hopper Crater}

The spatial resolution of the largest MASCS footprints inside Hopper is insufficient to discriminate the hollows from the crater floors (Figure 3). However, these observations can be used to investigate the evolution of the spectral parameters with the percentage of hollows material in the MASCS footprint (Table 3). We found a linear increase in the absolute reflectance at $750 \mathrm{~nm}$ with increasing the percentage of hollows in the footprint area. The same result is observed with the visible slope (Table 3 ). The orbit with the highest spatial resolution confirms this observation: the footprints covering 100 $\%$ of the hollows field show higher values of VIS-slope and R750 than the footprints which did not contain the hollows field (Table 4). The evolution of these parameters is independent of the observation conditions (viewing geometry, in particular emission angle, and temperature).

The increasing VIS-slope with the increasing percentage of hollows in the footprint is a surprising result. In fact, the hollows are described in literature as having a less steep slope than the surrounding material (Blewett et al., 2013; Thomas et al., 2016). However, in our study, the calculation of the slope in the visible seems impacted by the shape of the spectrum in the UV (see section 3.1). Although the Curvature is not linearly correlated with the percentage of hollows in the footprint, footprints containing $0 \%$ hollows still have lowest Curvature values. Furthermore, footprints with $100 \%$ hollows have the highest Curvature values (Table 3).

Table 2. Spectral parameters derived from the observations of the orbit with the largest footprints (ob2_12268_133540) in Hopper impact crater (Figure 3).

\begin{tabular}{cccccc}
\hline $\begin{array}{c}\text { Area of hollows } \\
\text { in footprint (\%) }\end{array}$ & $\mathbf{R 7 5 0}$ & VIS-slope** & UV-downturn & VISNIR-slope & Curvature \\
\hline $\mathbf{0}$ & 0.038 & 0.70 & 3.08 & 0.64 & 3.00 \\
\hline $\mathbf{2 5}$ & 0.048 & 0.77 & 3.06 & 0.64 & 5.04 \\
\hline $\mathbf{4 5}$ & 0.049 & 0.86 & 3.17 & 0.72 & 4.64 \\
\hline $\mathbf{8 0}$ & 0.064 & 0.89 & 3.19 & 0.66 & 8.20 \\
\hline $\mathbf{9 5}$ & 0.066 & 0.95 & 3.26 & 0.70 & 7.97 \\
\hline
\end{tabular}

*Pearson correlation coefficient between percentage of hollows in the footprint and R750: 0.99 (significance level: $99 \%$ ) $* *$ Pearson correlation coefficient between percentage of hollows in the footprint and VIS-slope: 0.98 (significance level: 97\%) 
Table 3. Spectral parameters obtained from the observations of the orbit with the smallest footprints (ob3_13276_093728) in Hopper impact crater (Figure 3).

\begin{tabular}{cccccc}
\hline $\begin{array}{c}\text { Area of hollows } \\
\text { in footprint (\%) }\end{array}$ & $\mathbf{R 7 5 0}$ & VIS-slope** & UV-downturn & VISNIR-slope & Curvature \\
\hline $\mathbf{0}$ & 0.038 & 0.72 & 3.01 & 0.72 & 2.72 \\
\hline $\mathbf{0}$ & 0.037 & 0.70 & 3.11 & 0.65 & 3.09 \\
\hline $\mathbf{0}$ & 0.037 & 0.68 & 3.12 & 0.59 & 2.75 \\
\hline $\mathbf{1 0 0}$ & 0.069 & 0.99 & 3.16 & 0.64 & 8.07 \\
\hline $\mathbf{1 0 0}$ & 0.069 & 1.01 & 3.23 & 0.63 & 8.72 \\
\hline $\mathbf{1 0 0}$ & 0.073 & 1.06 & 3.25 & 0.73 & 9.09 \\
\hline
\end{tabular}

*Pearson correlation coefficient between percentage of hollows in the footprint and R750: 0.99 (significance level: 99\%) ${ }^{* *}$ Pearson correlation coefficient between percentage of hollows in the footprint and VIS-slope: 0.98 (significance level: 97\%)

\section{Investigation of absorption bands in hollows}

Previous analysis of MASCS spectra did not identify any absorption features, suggesting that Mercury's surface is dominated by silicates with low ferrous iron content (Izenberg et al., 2014). Thomas et al. (2016) did not find any absorptions in the MASCS spectra obtained in the hollows of Eminescu and Raditladi. However, they found a weak absorption band in the MDIS data for Raditladi centered around $600 \mathrm{~nm}$, as observed in the Dominici, Hopper, Canova and Velazquez impact craters by Vilas et al. (2016) and Lucchetti et al. (2018). In this study, we performed a detailed analysis in order to investigate absorption features specific to hollows in the four studied samples.

\subsection{Searching absorption bands with MASCS}

A continuum removal has been applied to the MASCS spectra of hollows to highlight the absorption bands observed with MDIS between 528 and $828 \mathrm{~nm}$ with a maximum depth of 4\% (Vilas et al., 2016; Lucchetti et al., 2018) and towards $1000 \mathrm{~nm}$ (Lucchetti et al., 2018). The shape of the MASCS spectra of hollows in the visible is not linear (Figure 5), thus the continuum is approximated by a polynomial fit of degree 2 between 400 and $828 \mathrm{~nm}$, and a straight line between 828 and $1400 \mathrm{~nm}$. Continuum removed spectra are flat and lack absorption bands (Figure 9). The noise of the MASCS VIS detector is stable between 400 and $700 \mathrm{~nm}$ and seems to be related to the instrument. At longer wavelengths, the noise increases and differs between observations. A strong signal variation occurs between 800 and $900 \mathrm{~nm}$, and is not related to the junction of the spectra obtained by the 2 detectors (Besse et al., 2015). This small jump in reflectance is reported in spectra obtained in various units and is also observable on the Mercury's reference spectra obtained using more than 850000 spectra (Izenberg et al., 2014). We interpreted this variation in reflectance as a residual error in the calibration related to a change in the response of the instrument in this wavelength range. As expected, in the NIR the signalto-noise ratio decreases and no conclusion can be made about the existence of a weak absorption band towards $1000 \mathrm{~nm}$.

The large phase angles of the MASCS data could be the source of the lack of absorption bands in the MASCS spectra. However, Varatharajan et al. (2019) has shown 

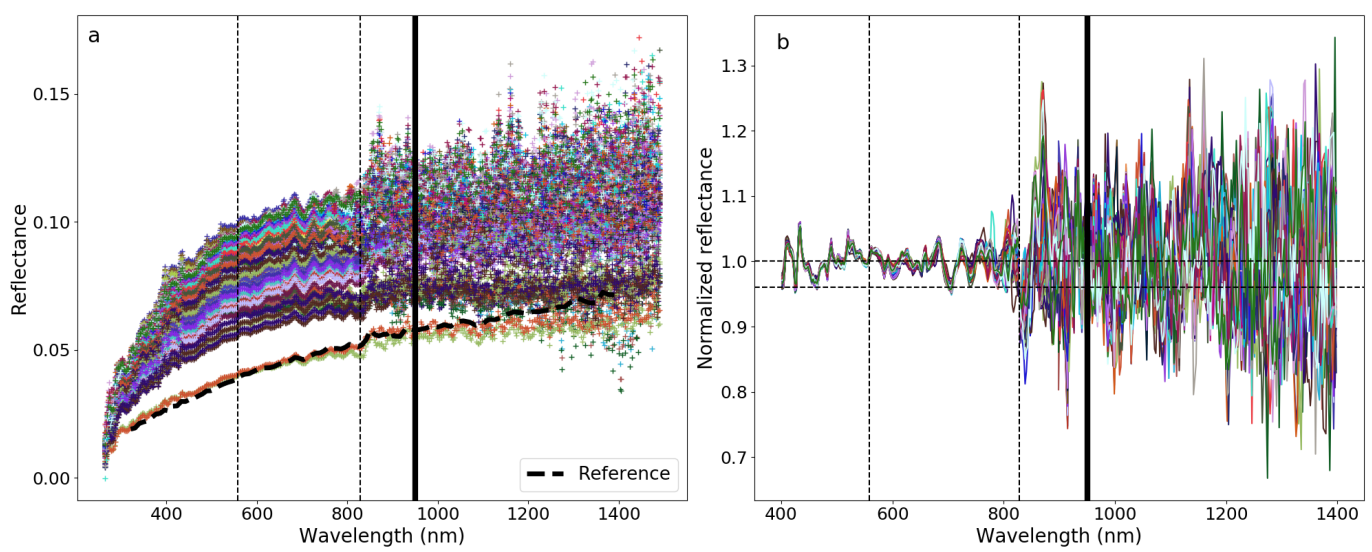

Figure 9. a) MASCS spectra obtained in hollows (113). The two spectra close to the reference spectrum are measured in Hopper impact crater and contain 25\% and $45 \%$ of hollows (cf. Table 2). b) Continuum removed hollows spectra. The thick vertical black line shows the junction of the two detectors of MASCS VIRS spectrometer. The two vertical dashed lines are the wavelengths used by Vilas et al. (2016) and Lucchetti et al. (2018) to define the absorption bands in MDIS filters. The horizontal dashed lines highlight a band depth of $4 \%$ as reported in Lucchetti et al. (2018). No absorptions above the noise are seen in these MASCS spectra.

that the phase angle has only minor effects on sulfides spectral features in the UV and VIS. Residual calibration error is a possible factor explaining the differences between the two instruments. For example, a calibration error could create an absorption band in MDIS or remove one in the MASCS data. Another hypothesis to explain the lack of absorption features is a low content of pure sulfides in the MASCS footprints. In fact, Izenberg et al. (2014) showed that at least $75 \%$ of pure sulfides are needed in the MASCS fieldof-view to be detectable in the spectra. The absorption band around $600 \mathrm{~nm}$ observed in several hollows has a maximum depth of $4 \%$ (Vilas et al., 2016; Lucchetti et al., 2018), if the concentration of pure sulfides is too low the band is within the noise of MASCS spectra.

However, the absence of a band at $600 \mathrm{~nm}$ does not exclude the presence of sulfides. Helbert et al. (2013) showed that the thermal processing at Mercury daytime temperature reduces the spectral contrast of the diagnostic features of sulfides around 600 $\mathrm{nm}$. In addition, recent laboratory measurements under MASCS observing conditions show that the sulfides (fresh or heated) proposed in section 3.2 (CaS, NaS and one MgS sample) to explain the high values of UV-downturn in hollows spectra, do not exhibit absorption bands in the visible range (Varatharajan et al., 2019).

\subsection{MDIS and MASCS comparison of Hopper's hollows}

Intrinsic change in the composition of the hollows could explain why we do not observe an absorption band in the spectra obtained by MASCS. Vilas et al. (2016) studied the western part of the group of hollows in Hopper impact crater while MASCS looked at the eastern one. In order to investigate possible spectral changes within Hopper, we compared MDIS observations in the two parts of the hollows. Moreover, we compared MDIS measurements with MASCS spectra directly. We used the Experiment data record (EDR) of MDIS-WAC (Hawkins et al., 2007), and applied a radiometric and photometric calibration (Hapke, 1981; Domingue et al., 2015; Bott et al., 2019). Average reflectance obtained in both areas (the original area studied by Vilas et al. (2016), and the area ob- 


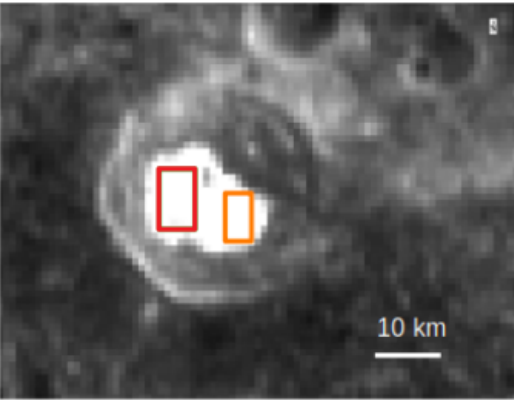

served with MASCS) of the hollows is represented in Figure 10. The absorption feature seems to be homogeneous in the hollows field from the MDIS observations, which excludes the hypothesis of an intrinsic spectral variation. The lack of spectral features in the MASCS spectra is then mostly likely linked to residual calibration errors in one of the two instruments or a pure sulfides concentration too low to be detected with the MASCS instrument (see section 4.1). Moreover, the absorption band observed by Vilas et al. (2016) in Hopper is the weakest of the hollows they studied. MASCS observations in the Dominici crater, where the band is deeper, do not have sufficient spatial resolution.

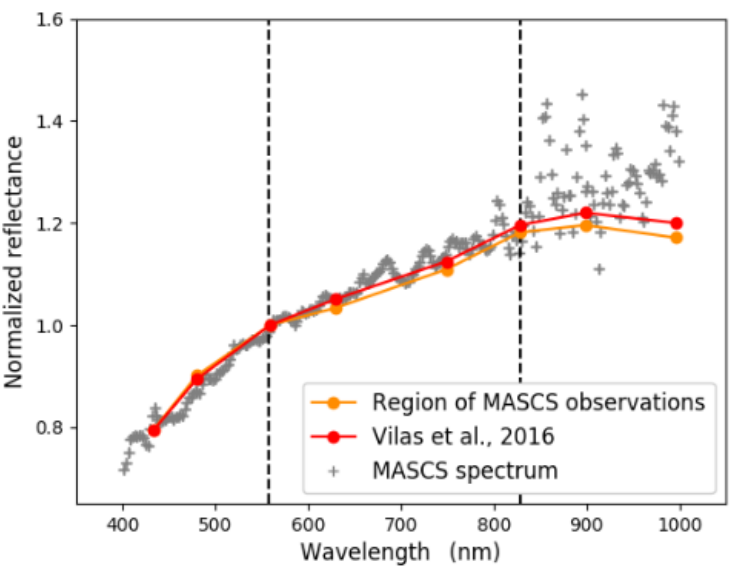

Figure 10. a) WAC-MDIS image (EW0211022288D) of Hopper crater with the red rectangle marking the area sampled by Vilas et al. (2016) and orange rectangle marking region observed with MASCS (Figure 3). b) Normalised reflectance of the two areas as observed by MDIS, and one example of normalized MASCS spectra in the same region. The two black dashed lines bound the upper and lower wavelength limits of the absorption band identified by Lucchetti et al. (2018) and Vilas et al. (2016). The aspect ratio is different from Vilas et al. (2016) however the depth of the absorption band is similar.

\section{Discussion}

\subsection{Origin of hollows volatile components}

\subsubsection{Are volatile components of hollows linked to pyroclastic deposits?}

The geological history of the Tyagaraja crater seems to be complex given that the crater floor is covered both by hollows and facula (Blewett et al., 2011). Unfortunately, the spatial resolution of images is insufficient to distinguish if the surface of the bright patches is fully covered by hollows material (Figure 3). Consequently, the spectral variability of Tyagaraja hollows could be due to different proportions of materials within the instrument footprint area (e.g. hollows, crater floor, facula). Tyagaraja's facula and crater floor have similar values of UV-downturn (Figure 11b). The hollows tend toward high UV downturns (Figure 11b). However, these units are clearly distinct in the Curvature and the VISNIR-slope (Figure 11c and d). As expected, the spectral slope of facula is redder than Mercury's average, while the spectral slope of hollows is generally less red. The Curvature parameter of hollows is much higher than the Curvature of the facula.

In section 3.1 and 3.2, we proposed several factors that can influence the spectral properties. These contributors can also influence the spectral properties of the pyroclastic deposits. If we considered the space weathering, resulting in a reddening of the spec- 
tral slope in the visible-near-infrared, as the only contributing factor for the VISNIR slope, the chronological sequence of events would therefore be: 1) formation of the facula, 2) crater formation and finally 3 ) the formation of the hollows. However, it is impossible for the facula to be older than the crater in which it formed. A physical difference (e.g. grain size) between each geological unit could play a role in the near-ultraviolet (Cloutis et al., 2008). However, space weathering or grain size is not known to create a curvature of the spectrum between 300 and $600 \mathrm{~nm}$ (see section 3.2). Compositional differences between each geological unit seem necessary to explain the different effects on the spectra.
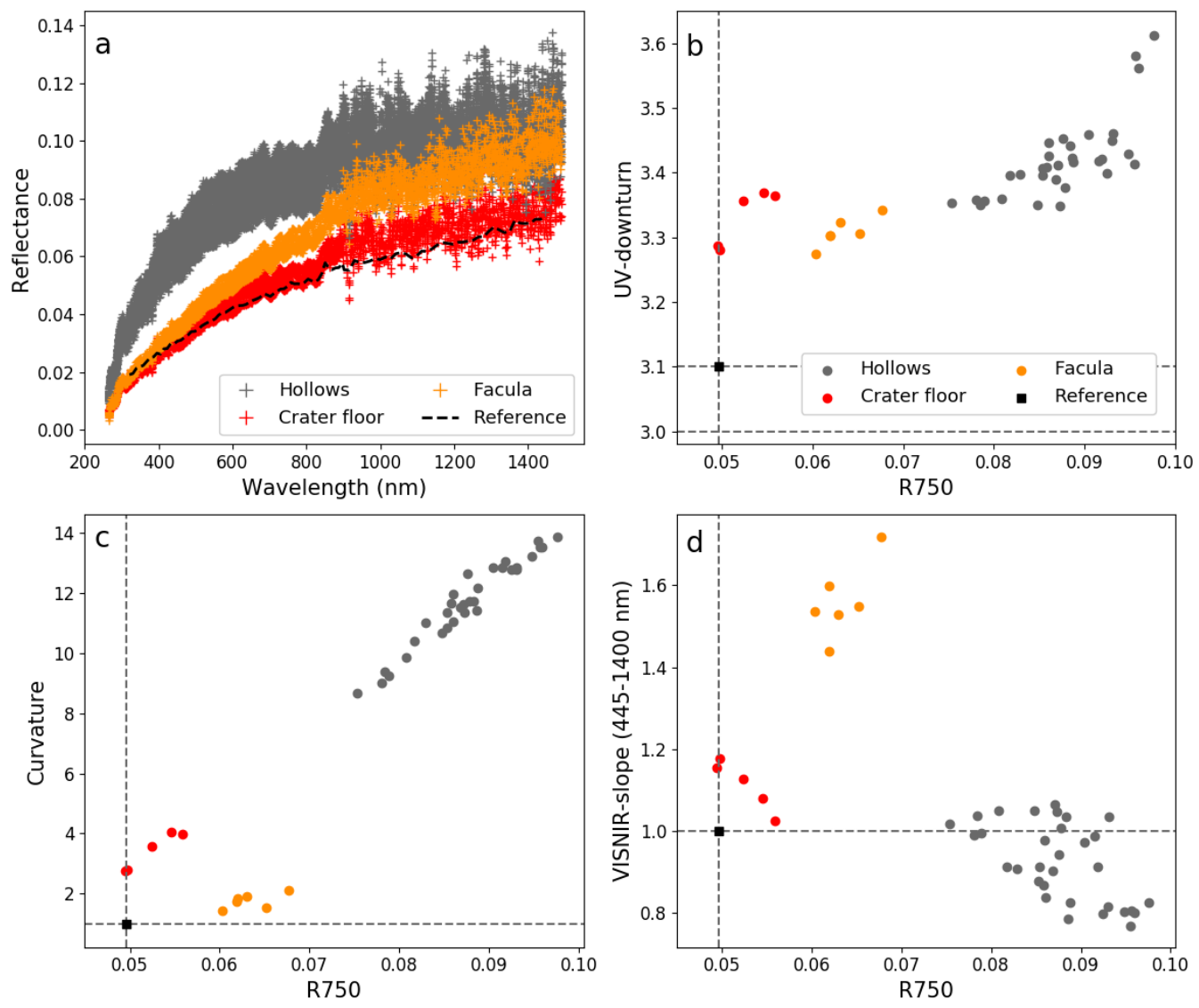

Figure 11. a) MASCS spectra obtained in the Tyagaraja impact crater for three geological units: facula, hollows and impact crater floor. Each spectra correspond to a footprint shown in Figure 2. b, c and d) Spectral parameters versus R750 for the spectra shown in (a). Hollows and facula in the Tyagaraja impact crater have distinct spectral properties.

Blewett et al. (2011, 2013) proposed that volatile species could be trapped under the pyroclastic deposits during the lava emplacement and after be at the origin of the hollows formation. Hollows formed under these conditions could have different properties than hollows formed on LRM or impact melt, for example, because the origin and/or nature of the volatile component could be different. However, in our study, the Tyagaraja hollows do not have spectral properties that are different from other hollows. In particular, they have spectral properties close to those of Warhol hollows (Figures 6, 7 and 8), yet no faculae are identified in Warhol impact crater. Thus, the volatile compound responsible for the formation of Tyagaraja's hollows is probably similar to the others. This result suggests two hypotheses: 
1. The material forming hollows has a close composition to volatile material that can be condensed or trapped after/during an explosive eruption on Mercury. Nittler et al. (2014) showed that the largest pyroclastic deposit on Mercury is depleted in S and C compared to their surroundings. These elements could be the volatile species driving the explosive eruptions on Mercury (Blewett et al., 2018) and also responsible for the formation of hollows.

2. The parent material of the hollows would be the crater floor, even if it is covered by faculae. In this case, the heat of the pyroclastic deposit on the impact crater floor could be responsible for the formation of the hollows, which could explain why hollows are often found near faculae. This hypothesis is supported by the differences in curvature and VISNIR-slope between faculae and hollows that suggest differences between material forming these units. Some faculae would have to be relatively young, as hollows are identified among the youngest formations on the surface of Mercury. Thomas et al. (2014a) showed that faculae occurred until less than $1 \mathrm{Ga}$, and Blewett et al. (2018) dated hollows as up to $1 \mathrm{Ga}$. Therefore, on a geological time scale, the formation of hollows and faculae could be close, supporting our hypothesis.

\subsubsection{Are volatile components of hollows linked to the host-crater?}

MASCS observations allow the comparison of the spectral properties between the hollows and their parent material: crater floor. We found a strong linear correlation between UV-downturn in the host-craters and their associated hollows (Figure 12). The same correlation is observed for the Curvature. This result is consistent with the correlation found before between UV-downturn and Curvature (see section 3.2). The spectral slopes are uncorrelated between hollows and the host crater floor.

This correlation could be explained by two hypotheses:

1. A mixing between hollows and local crater floor material in the MASCS footprints. We have shown in section 3.3 that a mixing can have an effect on spectral properties.

2. The physical and/or chemical properties of the hollows material are dependent on the parent material. If the volatile rich layer is created during the differentiation of the impact melt, as proposed by Vaughan et al. (2012), there is no reason for the composition of this layer to be the same for all the impact craters. In fact, the composition of the volatile-rich layer can vary for example with the depth of excavation, the material excavated and also with the temperature of the impact melt. The differentiation of a possible sulfide layer will therefore be different in terms of composition for each impact melt and crater, especially if heterogeneity is present in the crust even before the impact. Our analysis is in agreement with a different volatile layer depending on each impact crater. These interpretations exclude the presence of a global volatile rich layer with unique composition.

\subsection{Formation and evolution of hollows}

The high spatial resolution of MASCS observations and NAC-MDIS images around the central peak of Eminescu impact crater offers the opportunity to investigate the spectral properties across the different parts of this particular hollow (Figure 3). Some footprints are located in the interior of the hollows and others on the surrounding bright part. We found that all the parameters have higher values in the halo than in the hollows floor. In the VIS to NIR, the two parts of the hollows have less steep slopes than the impact crater floor (Figure 13). The UV-downturn in the hollows floor is close to the UV-downturn of the impact crater floor (Figure 13). The UV-downturn in the halo is greater than the UV-downturn in the host-crater. In the halo, all the parameters (VIS-slope, VISNIRslope, UV-downturn and curvature) are correlated with the R750, and we can observe 

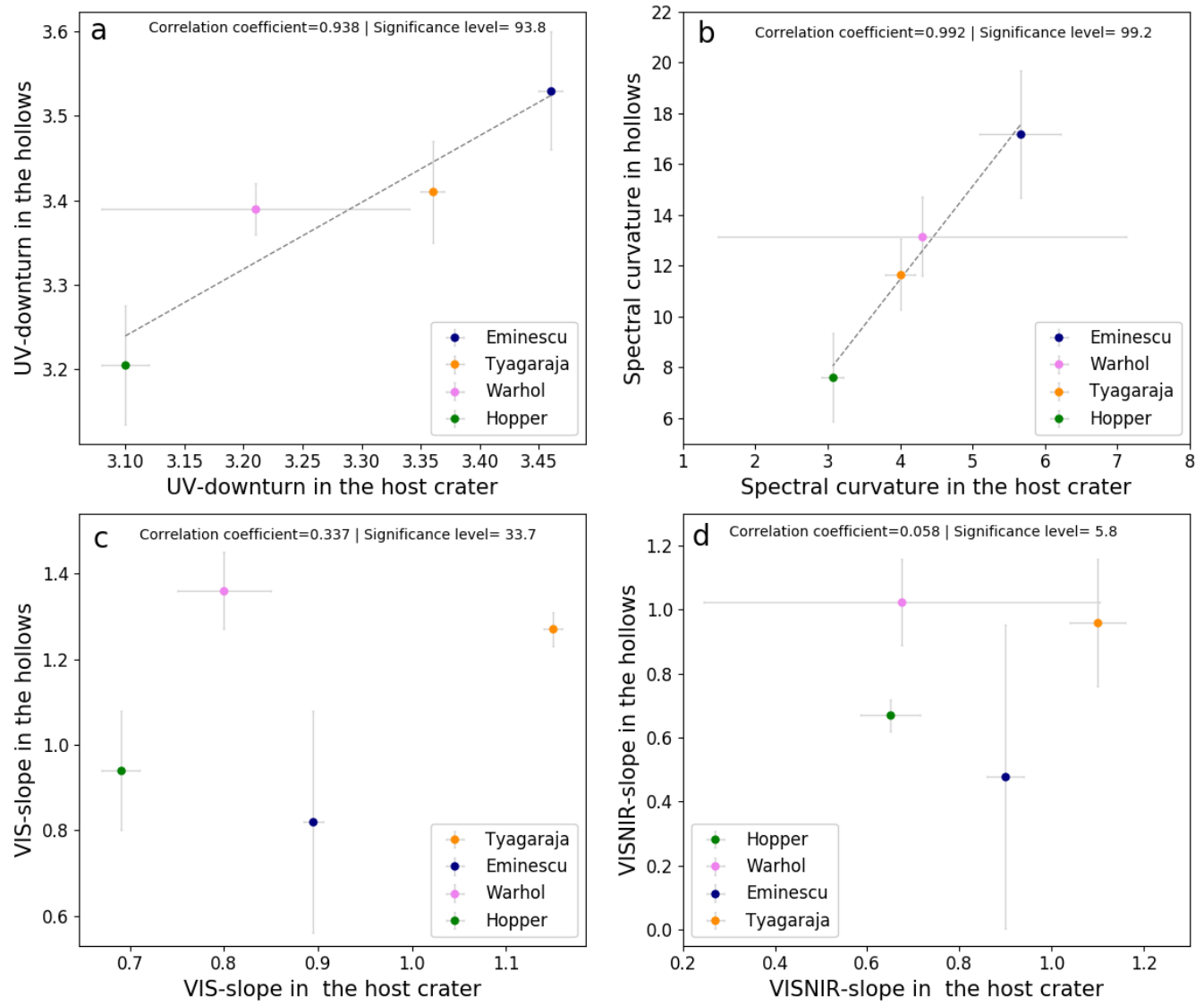

Figure 12. Linear correlation between parameters in the host crater and parameters of its associated hollows field. Dots represent the median value of all spectra used and the error bars correspond to the standard-deviation. Near-ultraviolet properties of hollows are dependent on the host crater.

a linear change between the hollows interior and the bright halo (Figure 13). Spectral parameters are not correlated to the observation conditions (incidence, emission, phase angles), so this evolution is not an artifact of varying viewing geometry.

Several hypotheses can explain this gradual change in spectral parameters between the two parts of the hollows: 1) The bright halo and the hollows interior have very different spectral properties, which at the spatial resolution of MASCS footprints results in spectra that are geographical mixtures between hollow floors material and halo material that create intermediate values of spectral parameters, 2) The bright halo tends gradually to have the same spectral properties of the hollows interior. The physical and chemical properties of the material can change along the halo from the inner part of it to the edges.

Blewett et al. (2013) proposed several explanations on the presence of the bright halo around hollows. Among these hypotheses are the destruction of nanophase sulfides and physical properties modified during the formation of the hollows. In this analysis, we showed that halo has also steeper slopes and higher curvature. The destruction of nanophase sulfides increases the reflectance and decreases the spectral slope and that could explain why hollows interior have higher reflectance and less steep VIS to NIR slope than parent material (crater floor). In addition, as mentioned in section 3.2, the particular 

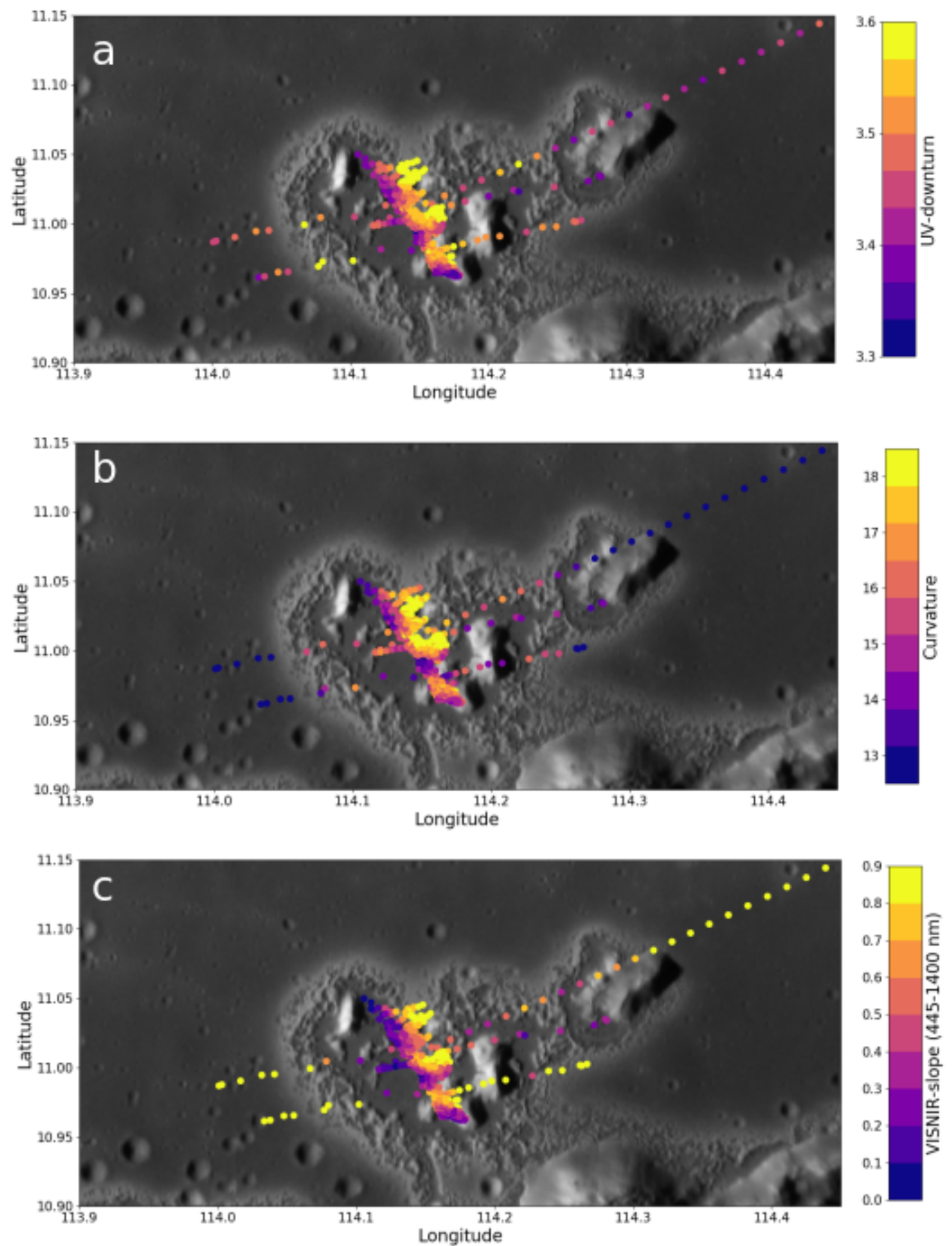

Figure 13. MASCS footprints in the Eminescu hollows (ob4_14327_010045,

ob4_14326_164731). The spatial resolution is from 0.3 to $1.7 \mathrm{~km} /$ footprint, so the length of the footprint is approximately four times larger than the dot. The color code shows increasing values of UV-downturn (a), curvature (b) and VISNIR-slope (c) from blue to yellow. The three parameters gradually increase between hollow floor and bright halo. 
tred around $300 \mathrm{~nm}$ associated with the sulfides CaS, $\mathrm{NaS}$ and MgS (Varatharajan et al., 2019). The results shown in Figure 13 are consistent with the loss of sulfides progressively decreasing the depth of the absorption band, and thus decreasing the UV-downturn and curvature during the hollows formation.

Moreover, the loss of a volatile component could result in a change in grain size. Thomas et al. (2016) showed differences in grain-size in Eminescu's hollows: grains seem to be finer in the halo than in the hollow floors. The reflectance increases when the grain size decreases (Crown \& Pieters, 1987). This result could explain the very high reflectance in the halo.

These observations support the scenario of a formation by scarp retreat proposed by Blewett et al. (2013). In fact, some favorable physical conditions could lead the formation process of hollows in a given spot. After the destruction of the volatile component, new material is exposed on the surface. The evolution of the reflectance spectra according to the growth of the hollow is represented in Figure 14. In the place where the hollows begins to grow, the reflectance, UV-downturn, and Curvature increases and the VISNIR-slope starts to slightly decrease (spectrum 3 compared to spectra 1, 2 in Figure 14a). This variation seems to be related to the beginning of the loss of the volatile element. At this point, unaltered material present under the regolith and rich in volatile elements seems to be exposed at the surface. Then, as the volatile element is destroyed in the unaltered material exposed, the reflectance, UV-downturn and Curvature begin to decrease (Spectrum 2 in figure 14c). The slope of the spectrum continues to decrease. The destruction of volatile-rich material changes the physical structure of the material exposed on the surface and makes it unstable. Some parts begin to destabilize and then collapse along the hollow escarpment exposing unaltered material on the surface. When the volatile phase is lost, the vertical progression of the hollows stops.

Determination of the nature and rate of hollows formation process is one of the objectives of the BepiColombo mission to Mercury (Rothery et al., 2020). In particular, VIHI (Visual and Infrared Hyperspectral Imager) on the instrumental suite SIMBIOSYS (Spectrometer and Imaging for MPO BepiColombo Integrated Observatory SYStem, Flamini et al., 2010; Cremonese et al., 2020) should be able to confirm the validity of the scenario proposed in figure 14. The spatial resolution of this instrument (up to $100 \mathrm{~m} / \mathrm{pixel}$ ) is represented on the figure 14 panel c. Observations of hollows with VIHI will certainly make it possible to define the spectral characteristics of each hollows facies (floor, bright halo and background terrain) more distinctly than the MASCS instrument and increase the number of hollows observed with sufficient spatial resolution to resolve each facies. 
a
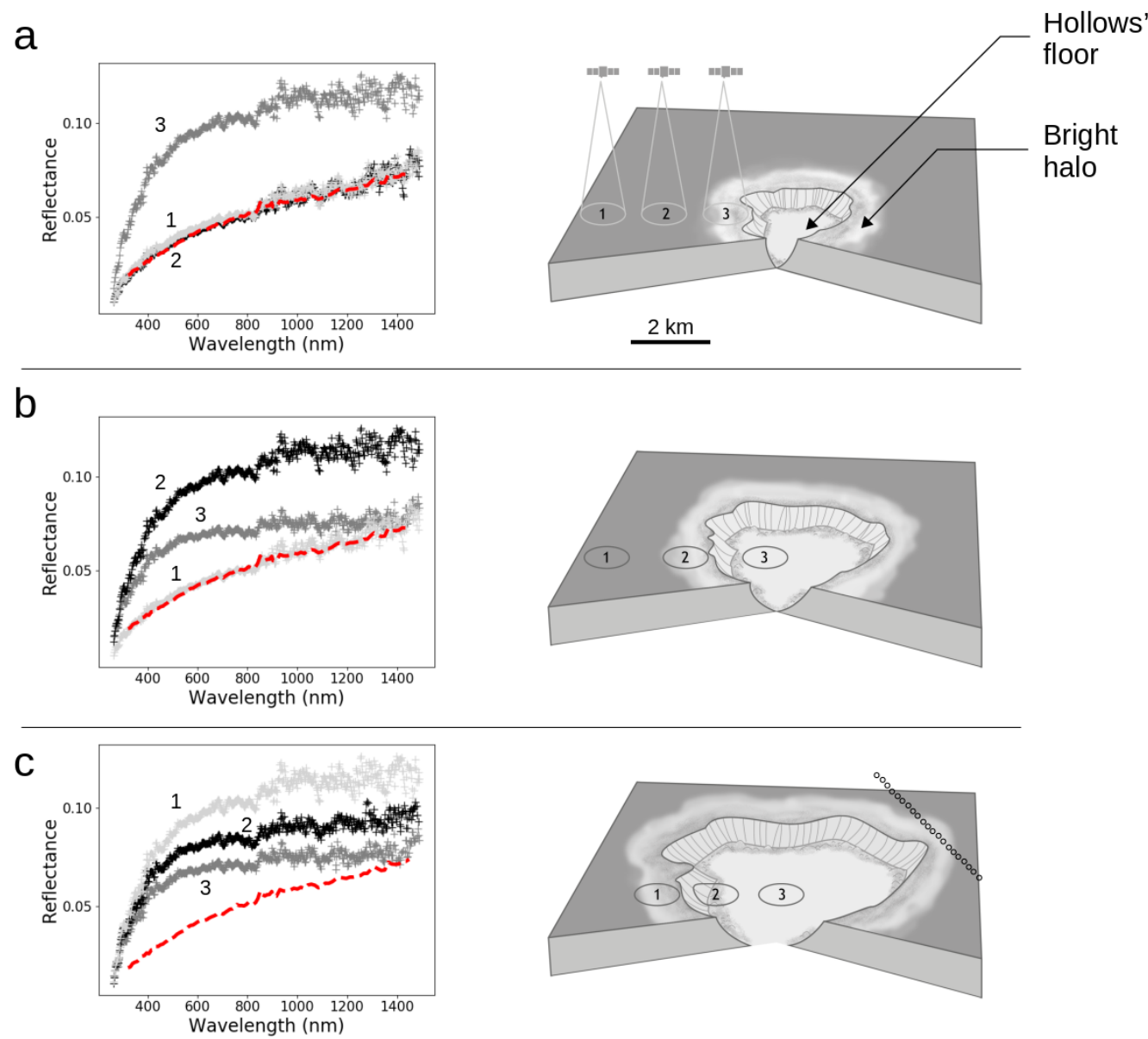

Figure 14. A schematic illustration of possible hollows growth and the evolution of reflectance spectra. Diagrams a, b and c are in chronological order. The red spectrum is the Mercury's reference (Izenberg et al., 2014). The small circles on panel c represent the spatial resolution of future hyper-spectral observations with BepiColombo.

\section{Conclusion}

Images from MESSENGER revealed the presence of hollows on the surface of Mercury; however their origin and nature remained unconstrained. Multispectral data showed that hollows are among the brightest features on the surface of Mercury and exhibit possible absorption features associated with sulfides. From the highest spatial resolution observations with the Mercury Atmospheric and Surface Composition spectrometer (MASCS) examined here, several important conclusions about the hollows can be made.

1. Hollows have unique spectral properties in the near-ultraviolet. Their reflectance spectra are quite distinct from other geological units. Hollows have a steeper downturn in reflectance between 300 and $350 \mathrm{~nm}$ and are characterized by a distinct spectral curvature between 300 and $600 \mathrm{~nm}$. These properties may be related to the absorption feature of certain sulfides such as $\mathrm{CaS}, \mathrm{NaS}$ and $\mathrm{MgS}$, centered around $300 \mathrm{~nm}$ (Varatharajan et al., 2019).

2. Hollows reflectance spectra exhibit no clear evidence of absorption features in the visible in MASCS data as was observed in the MDIS multi-color data. The lack of consistent absorption bands seems to be related to calibration errors in one or 
both instruments, or could be attributed a lack of sufficient concentration of sulfides in the MASCS field-of-view.

3. The evolution of spectral properties throughout the Eminescu hollows are consistent with a formation by destruction of nanophase sulfides. In addition, the spectral properties are correlated with the morphology of the hollows supporting the suggestion that hollows grow by scarp retreat.

4. Faculae and hollows have distinct spectral properties. This suggests that the volatile species driving explosive eruption and formation of hollows on Mercury have different nature and origin. On the other hand, near-ultraviolet and Curvature properties of hollows are correlated with properties of host crater floor implying that the composition and/or physical properties of the hollows material depend on those of the host crater. This supports the hypothesis of the differentiation of a volatile rich layer in the impact melt.

The Bepicolombo mission is equipped with an instrumental suite (Flamini et al., 2010; Cremonese et al., 2020) including a hyperspectral imager: VIHI (Visual and Infrared Hyperspectral Imager). This instrument has higher spatial resolution (up to 100 $\mathrm{m} /$ pixel), a higher signal-to-noise ratio and a wider range of wavelength (0.4 to $2 \mu \mathrm{m}$ ) than MASCS. The future VIHI global coverage (at $480 \mathrm{~m} /$ pixel) and high resolution images (which will cover $20 \%$ of the surface) will extend the MASCS results obtained in hollows (Cremonese et al., 2020). These observations of different hollows will provide additional insight into the nature and processes of hollow formation. In addition, these data will be essential in differentiating the effects of composition, grain size and space-weathering on hollow spectra. Together with observations from the spectrometer MERTIS (Mercury Radiometer and Thermal Infrared Spectrometer, Hiesinger et al., 2010), BepiColombo may lead to the discovery of new spectral characteristics of hollows at infrared wavelengths (7 to $14 \mu \mathrm{m})$ not observed by MASCS.

\section{Acknowledgments}

The authors acknowledge the Centre National des Etudes Spatiales (CNES) for continuous and long term support. O. Barraud acknowledges the support of the European Space Astronomy Centre (ESAC) faculty council for funding a visit to ESAC as part of this work. J. M. Sunshine was a visiting scientist at LESIA -Observatoire de Paris during this study. Special thanks to Sylvain Cnudde for the drawing in Figure 14. The data used in this work are available at the PDS Geosciences Node of Washington University, St. Louis, USA. The data set https://pds-geosciences.wustl.edu/messenger/mess-e_v_h-mascs3-virs-cdr-caldata-v1/messmas_2101/data/ddr/ was used which contains the latest calibration provided by the MESSENGER science team. The authors thank the reviewers for their constructive reviews and Jasinghege Don P. Deshapriya for his careful proofreading.

\section{References}

Bennett, C. J., McLain, J. L., Sarantos, M., Gann, R. D., DeSimone, A., \& Orlando, T. M. (2016). Investigating potential sources of mercury's exospheric calcium: Photon-stimulated desorption of calcium sulfide. Journal of Geophysical Research: Planets, 121(2), 137-146. doi: 10.1002/2015JE004966

Besse, S., Doressoundiram, A., Barraud, O., Griton, T., L. Cornet, Mũnnoz Crego, C., Varatharajan, I., \& Helbert, J. (2020). Spectral properties and physical extent of pyroclastic deposits on mercury: Variability within selected deposits and implications for explosive volcanism,. Journal of Geophysical Research: Planets. doi: 10.1029/2018JE005879

Besse, S., Doressoundiram, A., \& Benkhoff, J. ～(2015). S Spectroscopic properties of explosive volcanism within the caloris basin with messenger observa- 
tions. Journal of Geophysical Research: Planets, 120(12), 2102-2117. doi: 10.1002/2015JE004819

Blewett, D. T., Chabot, N. L., Denevi, B. W., Ernst, C. M., Head, J. W., Izenberg, N. R., .. Hurwitz, D. M. (2011). Hollows on mercury: Messenger evidence for geologically recent volatile-related activity. Science, 333(6051), 1856-1859. doi: $10.1126 /$ science.1211681

Blewett, D. T., Ernst, C. M., Murchie, S. L., \& Vilas, F. (2018). Mercury's hollows. In S. C. Solomon, L. R. Nittler, \& B. J. Anderson (Eds.), Mercury:the view after messenger (p. 1-29). Cambridge, U.K.: Cambridge University Press.

Blewett, D. T., Levy, C. L., Chabot, N. L., Denevi, B. W., Ernst, C. M., \& Murchie, S. L. (2014). Phase-ratio images of the surface of mercury: Evidence for differences in sub-resolution texture. Icarus, 242, 142 - $148 . \quad$ doi: https://doi.org/10.1016/j.icarus.2014.08.024

Blewett, D. T., Vaughan, W. M., Xiao, Z., Chabot, N. L., Denevi, B. W., Ernst, C. M., ... Solomon, S. C. (2013). Mercury's hollows: Constraints on formation and composition from analysis of geological setting and spectral reflectance. Journal of Geophysical Research: Planets, 118(5), 1013-1032. doi: 10.1029/2012JE004174

Bott, N., Doressoundiram, A., Zambon, F., Carli, C., Guzzetta, L., Perna, D., \& Capaccioni, F. (2019). Global spectral properties and lithology of mercury: The example of the shakespeare (h-03) quadrangle. Journal of Geophysical Research: Planets, 124(9), 2326-2346. doi: 10.1029/2019JE005932

Byrne, P. K., Whitten, J. L., Klimczak, C., McCubbin, F. M., \& Ostrach, L. R. (2018). The volcanic character of mercury. In S. C. Solomon, L. R. Nittler, \& B. J. Anderson (Eds.), Mercury:the view after messenger (p. 1-29). Cambridge, U.K.: Cambridge University Press.

Cloutis, E. A., McCormack, K. A., Bell, J. F., Hendrix, A. R., Bailey, D. T., Craig, M. A., .. Riner, M. A. (2008). Ultraviolet spectral reflectance properties of common planetary minerals. Icarus, 197(1), $321-347 . \quad$ doi: https://doi.org/10.1016/j.icarus.2008.04.018

Cremonese, G., Capaccioni, F., Capria, M. T., Doressoundiram, A., Palumbo, P., Vincendon, M., ... Turrini, D. (2020, June). SIMBIO-SYS: Scientific Cameras and Spectrometer for the BepiColombo Mission. , 216(5), $75 . \quad$ doi: 10.1007/s11214-020-00704-8

Crown, D. A., \& Pieters, C. M. (1987). Spectral properties of plagioclase and pyroxene mixtures and the interpretation of lunar soil spectra. Icarus, 72(3), 492 506.

Denevi, B. W., Chabot, N. L., Murchie, S. L., Becker, K. J., Blewett, D. T., Domingue, D. L., ... Solomon, S. C. (2018). Calibration, projection, and final image products of messenger's mercury dual imaging system. Space Science Reviews, 214(1). doi: 10.1007/s11214-017-0440-y

Denevi, B. W., Ernst, C. M., Meyer, H. M., Robinson, M. S., Murchie, S. L., Whitten, J. L., .. Peplowski, P. N. (2013). The distribution and origin of smooth plains on mercury. Journal of Geophysical Research: Planets, 118(5), 891-907. doi: $10.1002 /$ jgre.20075

Denevi, B. W., Robinson, M. S., Solomon, S. C., Murchie, S. L., Blewett, D. T., Domingue, D. L., ... Chabot, N. L. (2009). The evolution of mercury's crust: A global perspective from messenger. Science, 324(5927), 613-618.

Domingue, D. L., Chapman, C. R., Killen, R. M., Zurbuchen, T. H., Gilbert, J. A., Sarantos, M., ... McClintock, W. E. (2014). Mercury's WeatherBeaten Surface: Understanding Mercury in the Context of Lunar and Asteroidal Space Weathering Studies. Space Sci Rev, 181, 121-214. doi: 10.1007/s11214-014-0039-5

Domingue, D. L., Hash, C. D., Denevi, B. W., \& Murchie, S. L. (2017). Extending messenger's mercury dual imager's eight-color photometric standardization to 
cover all eleven filters. Icarus, 297, 83 - 89. doi: https://doi.org/10.1016/ j.icarus.2017.06.023

Domingue, D. L., Murchie, S. L., Denevi, B. W., Ernst, C. M., \& Chabot, N. L. (2015). Mercury's global color mosaic: An update from messenger's orbital observations. Icarus, 257, 477 - 488. doi: https://doi.org/10.1016/ j.icarus.2014.11.027

Fischer, E. M., \& Pieters, C. M. (1994, October). Remote Determination of Exposure Degree and Iron Concentration of Lunar Soils Using VIS-NIR Spectroscopic Methods. , 111(2), 475-488. doi: 10.1006/icar.1994.1158

Flamini, E., Capaccioni, F., Colangeli, L., Cremonese, G., Doressoundiram, A., Josset, J., ... others (2010). Simbio-sys: The spectrometer and imagers integrated observatory system for the bepicolombo planetary orbiter. Planetary and Space Science, 58(1-2), 125-143.

Goudge, T. A., Head, J. W., Kerber, L., Blewett, D. T., Denevi, B. W., Domingue, D. L., ... Solomon, S. C. (2014). Global inventory and characterization of pyroclastic deposits on mercury: New insights into pyroclastic activity from messenger orbital data. Journal of Geophysical Research: Planets, 119(3), 635-658. doi: 10.1002/2013JE004480

Hapke, B. (1981). Bidirectional reflectance spectroscopy: 1. theory. Journal of Geophysical Research: Solid Earth, 86(B4), 3039-3054. 일.1029/ JB086iB04p03039

Hawkins, S., Boldt, J., Darlington, E., Espiritu, R., Gold, R., Gotwols, B., ... Williams, B. (2007). The mercury dual imaging system on the messenger spacecraft. Space Science Reviews, 131(1-4), 247-338. doi: 10.1007/ s11214-007-9266-3

Helbert, J., Maturilli, A., \& D'Amore, M. (2013). Visible and near-infrared reflectance spectra of thermally processed synthetic sulfides as a potential analog for the hollow forming materials on mercury. Earth and Planetary Science Letters, 369-370, 233 - 238. doi: https://doi.org/10.1016/j.epsl.2013.03.045

Hendrix, A. R., \& Vilas, F. (2006). The Effects of Space Weathering at UV Wavelengths: S-Class Asteroids. The Astronomical Journal, 132(3), 1396-1404. doi: $10.1086 / 506426$

Hiesinger, H., Helbert, J., \& Team, M. C.-I. (2010). The mercury radiometer and thermal infrared spectrometer (mertis) for the bepicolombo mission. Planetary and Space Science, 58(1-2), 144-165.

Izenberg, N. R., \& Holsclaw, G. M. (2017). New Ultraviolet Through Near Infrared Surface Reflectance Data Products from MESSENGER. In Lunar and planetary science conference.

Izenberg, N. R., Klima, R. L., Murchie, S. L., Blewett, D. T., Holsclaw, G. M., McClintock, W. E., ... Dyar, M. D. (2014). The low-iron, reduced surface of mercury as seen in spectral reflectance by messenger. Icarus, 228, $364-374$. doi: https://doi.org/10.1016/j.icarus.2013.10.023

Izenberg, N. R., Thomas, R. J., Blewett, D. T., \& Nittler, L. R. (2015). Are there compositionally different types of hollows on mercury? In Lunar and planetary science conference.

Kerber, L., Head, J. W., Blewett, D. T., Solomon, S. C., Wilson, L., Murchie, S. L., ... Domingue, D. L. (2011). The global distribution of pyroclastic deposits on mercury: The view from messenger flybys 1-3. Planetary and Space Science, 59(15), 1895 - 1909. (Mercury after the MESSENGER flybys) doi: https://doi.org/10.1016/j.pss.2011.03.020

Kerber, L., Head, J. W., Solomon, S. C., Murchie, S. L., Blewett, D. T., \& Wilson, L. (2009). Explosive volcanic eruptions on mercury: Eruption conditions, magma volatile content, and implications for interior volatile abundances. Earth and Planetary Science Letters, 285(3), 263 - 271. (MESSENGER's First Flyby of Mercury) doi: https://doi.org/10.1016/j.epsl.2009.04.037 
Lucchetti, A., Pajola, M., Galluzzi, V., Giacomini, L., Carli, C., Cremonese, G., ... Palumbo, P. (2018). Mercury hollows as remnants of original bedrock materials and devolatilization processes: A spectral clustering and geomorphological analysis. Journal of Geophysical Research: Planets, 123(9), 2365-2379. doi: 10.1029/2018JE005722

McClintock, W. E., Izenberg, N. R., Holsclaw, G. M., Blewett, D. T., Domingue, D. L., Head, J. W., .. Vilas, F. (2008). Spectroscopic observations of mercury's surface reflectance during messenger's first mercury flyby. Science, 321 (5885), 62-65. doi: 10.1126/science.1159933

McClintock, W. E., \& Lankton, M. R. (2007). The mercury atmospheric and surface composition spectrometer for the messenger mission. Space Science Reviews, 131(1-4), 481-521. doi: 10.1007/s11214-007-9264-5

McCord, T. B., \& Adams, J. B. (1972a). Mercury: Surface Composition from the Reflection Spectrum. Science, 178(4062), 745-747. doi: 10.1126/science.178 .4062 .745

McCord, T. B., \& Adams, J. B. (1972b). Mercury: Interpretation of optical observations. Icarus, 17(3), 585 - 588. doi: https://doi.org/10.1016/0019-1035(72) 90024-3

Nittler, L. R., Starr, R. D., Weider, S. Z., McCoy, T. J., Boynton, W. V., Ebel, D. S., ... Sprague, A. L. (2011). The major-element composition of mercury's surface from messenger x-ray spectrometry. Science, 333(6051), 1847-1850. doi: $10.1126 /$ science. 1211567

Nittler, L. R., Weider, S., Starr, R., Chabot, N., Denevi, B., Ernst, C., ... others (2014). Sulfur-depleted composition of mercury's largest pyroclastic deposit: Implications for explosive volcanism and surface reflectance on the innermost planet. In Lunar and planetary science conference (Vol. 45, p. 1391).

Robinson, M. S., Murchie, S. L., Blewett, D. T., Domingue, D. L., Hawkins, S. E., Head, J. W., ... Watters, T. R. (2008). Reflectance and color variations on mercury: Regolith processes and compositional heterogeneity. Science, 321 (5885), 66-69. doi: 10.1126/science.1160080

Rothery, D. A., Massironi, M., Alemanno, G., Barraud, O., Besse, S., Bott, N., ... Zambon, F. (2020). Rationale for BepiColombo Studies of Mercury's Surface and Composition. , 216(4), 66. doi: 10.1007/s11214-020-00694-7

Solomon, S. C., McNutt, R. L., Gold, R. E., \& Domingue, D. L. (2007). Messenger mission overview. Space Science Reviews, 131(1-4), 3-39. doi: 10.1007/s11214 $-007-9247-6$

Thomas, R. J., Hynek, B. M., Rothery, D. A., \& Conway, S. J. (2016). Mercury's low-reflectance material: Constraints from hollows. Icarus, 277, 455 - 465. doi: https://doi.org/10.1016/j.icarus.2016.05.036

Thomas, R. J., Rothery, D. A., Conway, S. J., \& Anand, M. (2014a). Hollows on mercury: Materials and mechanisms involved in their formation. Icarus, 229, 221 - 235. doi: https://doi.org/10.1016/j.icarus.2013.11.018

Varatharajan, I., Maturilli, A., Helbert, J., Alemanno, G., \& Hiesinger, H. (2019). Spectral behavior of sulfides in simulated daytime surface conditions of mercury: Supporting past (messenger) and future missions (bepicolombo). Earth and Planetary Science Letters, 520, 127 - 140. $\quad$ doi: https://doi.org/10.1016/ j.epsl.2019.05.020

Vaughan, W. M., Helbert, J., Blewett, D. T., Head, J. W., Murchie, S. L., Gwinner, K., ... Solomon, S. C. (2012). Hollow-Forming Layers in Impact Craters on Mercury: Massive Sulfide or Chloride Deposits Formed by Impact Melt Differentiation? In Lunar and planetary science conference (p. 1187).

Vilas, F., Domingue, D. L., Helbert, J., D'Amore, M., Maturilli, A., Klima, R. L., ... Head, J. W. (2016). Mineralogical indicators of mercury's hollows composition in messenger color observations. Geophysical Research Letters, 43(4), 1450-1456. doi: 10.1002/2015GL067515 
Vilas, F., \& Hendrix, A. R. (2015). The UV/Blue Effects of Space Weathering Manifested in S-Complex Asteroids. I. Quantifying Change with Asteroid Age. , 150(2), 64. doi: 10.1088/0004-6256/150/2/64

Xiao, Z., Strom, R. G., Blewett, D. T., Byrne, P. K., Solomon, S. C., Murchie, S. L., ... Helbert, J. (2013). Dark spots on mercury: A distinctive low-reflectance material and its relation to hollows. Journal of Geophysical Research: Planets, 118(9), 1752-1765. doi: 10.1002/jgre.20115 